\title{
Ramification theory in non-abelian local class field theory
}

\author{
by \\ KÂZim Ilhan Ikeda and Erol Serbest (Istanbul) \\ To the memory of I. M. Gelfand
}

1. Introduction. Let $K$ be a local field, that is, a complete discrete valuation field with finite residue class field $\kappa_{K}$ of $q=p^{f}$ elements. For technical reasons, throughout the paper we shall assume that the multiplicative group $\boldsymbol{\mu}_{p}\left(K^{\text {sep }}\right)$ of all $p$ th roots of unity in $K^{\text {sep }}$ satisfies $\boldsymbol{\mu}_{p}\left(K^{\text {sep }}\right) \subset K$. Fix a Lubin-Tate splitting $\varphi$ over $K$. That is, we fix an extension $\varphi$ of the Frobenius automorphism of $K^{\mathrm{nr}}$ to $K^{\mathrm{sep}}$ (for details, cf. [Ko-dS]). In a sequence of papers [Ik-Se-1, Ik-Se-2, Ik-Se-3, following the idea of Fesenko developed in [Fes-1, Fes-2, Fes-3], we have constructed the non-abelian local reciprocity map $\boldsymbol{\Phi}_{K}^{(\varphi)}$ for $K$, which is an isomorphism from the absolute Galois group $G_{K}$ of $K$ onto a certain topological group $\nabla_{K}^{(\varphi)}$ which depends on the choice of the Lubin-Tate splitting $\varphi$.

The aim of the present paper is to study the ramification-theoretic properties of the map $\boldsymbol{\Phi}_{K}^{(\varphi)}$. We prove (in Theorems 4.15 and 4.16 that $\boldsymbol{\Phi}_{K}^{(\varphi)}$ is compatible with the refined higher ramification "filtration" of the absolute Galois group $G_{K}$ of $K$ (cf. 4.1 and the refined "filtration" of $\nabla_{K}^{(\varphi)}$ (cf. 4.2 ).

The organization of the paper is as follows. In Section 2, we collect the necessary results from the theory of local fields. In Section 3, we briefly review the main results of [Ik-Se-2] on the generalized Fesenko reciprocity map, and then sketch the construction of the non-abelian local reciprocity $\operatorname{map} \boldsymbol{\Phi}_{K}^{(\varphi)}$ following $[\underline{\mathrm{Ik}-\mathrm{Se}-3}$. In the last section, we first introduce the refined filtrations on $G_{K}$ and on $\nabla_{K}^{(\varphi)}$ and then prove the main results of the paper, which are stated as Theorems 4.15 and 4.16 .

2010 Mathematics Subject Classification: Primary 11S37.

Key words and phrases: local fields, higher-ramification theory, APF-extensions, FontaineWintenberger fields of norms, generalized Fesenko reciprocity map, non-abelian local reciprocity map, non-abelian local class field theory. 
2. Preliminaries on local fields. In this section, we shall briefly review the necessary background material from the theory of local fields.

2.1. Local fields. Throughout this work, $K$ will denote a local field, that is, a complete discrete valuation field with finite residue class field $O_{K} / \mathfrak{p}_{K}=: \kappa_{K}$ of $q_{K}=q=p^{f}$ elements with $p$ a prime number, where $O_{K}$ denotes the ring of integers in $K$ with the unique maximal ideal $\mathfrak{p}_{K}$. Let $\boldsymbol{\nu}_{K}$ denote the corresponding normalized valuation on $K$ (normalized by $\boldsymbol{\nu}_{K}\left(K^{\times}\right)=\mathbb{Z}$ ). As usual, the unit group of $K$ is denoted by $U_{K}$ and the $i$ th higher unit group of $K$ by $U_{K}^{i}$, where $0 \leq i \in \mathbb{Z}$.

Let $K^{\text {sep }}$ denote a fixed separable closure of $K$, and $K^{\mathrm{nr}}$ the maximal unramified extension of $K$ inside $K^{\text {sep }}$. The unique extension of $\boldsymbol{\nu}_{K}$ to $K^{\text {sep }}$ will be denoted by $\widetilde{\boldsymbol{\nu}}$, and for any sub-extension $L / K$ of $K^{\mathrm{sep}} / K$, the normalized form of the valuation $\left.\widetilde{\boldsymbol{\nu}}\right|_{L}$ on $L$ will be denoted by $\boldsymbol{\nu}_{L}$. The completion of $K^{\mathrm{nr}}$ with respect to the valuation $\boldsymbol{\nu}_{K}$ nr will be denoted by $\widetilde{K}$. For any separable extension $L / K$, we put $\widetilde{L}:=L \widetilde{K}$.

Let $G_{K}$ denote the absolute Galois group $\operatorname{Gal}\left(K^{\text {sep }} / K\right)$. The topological generator of $\operatorname{Gal}\left(K^{\mathrm{nr}} / K\right)$, which is the Frobenius automorphism of $K$, is denoted by $\varphi_{K}=\varphi$ (if there is no risk of confusion). Any extension of the automorphism $\varphi: K^{\mathrm{nr}} \rightarrow K^{\mathrm{nr}}$ to $K^{\mathrm{sep}}$ is called a Lubin-Tate splitting over $K$ and is again denoted by $\varphi$.

We further assume that the multiplicative group $\boldsymbol{\mu}_{p}\left(K^{\mathrm{sep}}\right)$ of $p$ th roots of unity in $K^{\text {sep }}$ satisfies

$$
\boldsymbol{\mu}_{p}\left(K^{\mathrm{sep}}\right) \subset K .
$$

2.2. Local Artin reciprocity map. Let $G_{K}^{\text {ab }}$ denote the maximal abelian Hausdorff quotient group $G_{K} / G_{K}^{\prime}$ of the topological group $G_{K}$, where $G_{K}^{\prime}$ denotes the closure of the first commutator subgroup $\left[G_{K}, G_{K}\right]$ of $G_{K}$.

Recall that abelian local class field theory for the local field $K$ establishes a unique natural algebraic and topological isomorphism

$$
\operatorname{Art}_{K}: G_{K}^{\mathrm{ab}} \stackrel{\sim}{\longrightarrow} \widehat{K^{\times}},
$$

called the local Artin reciprocity map of $K$, where the topological group $\widehat{K^{\times}}$ denotes the pro-finite completion of the multiplicative group $K^{\times}$, satisfying certain properties. In particular, for an abelian extension $L / K$, and for every integer $0 \leq i \in \mathbb{Z}$ and real number $\nu \in(i-1, i]$,

$$
x \in U_{K}^{i} \mathcal{N}_{L} \Leftrightarrow \operatorname{Art}_{L / K}^{-1}(x) \in \operatorname{Gal}(L / K)^{\nu},
$$

where $x \in \widehat{K^{\times}}$. Here, $\mathcal{N}_{L}$ denotes the closed subgroup of $\widehat{K^{\times}}$defined to be the intersection $\mathcal{N}_{L}=\bigcap_{E} N_{E / K} \widehat{E^{\times}}$, where $E$ runs over all finite extensions of $K$ inside $L$. 
In what follows, we shall briefly review the higher ramification subgroups in the upper numbering of the absolute Galois group $G_{K}$ of $K$.

2.3. A brief review of ramification theory. The main reference that we follow closely here is [Ik-Se-1].

For a finite separable extension $L / K$, and for any $\sigma \in \operatorname{Hom}_{K}\left(L, K^{\mathrm{sep}}\right)$, introduce

$$
i_{L / K}(\sigma):=\min _{x \in O_{L}}\left\{\boldsymbol{\nu}_{L}(\sigma(x)-x)\right\}
$$

put

$$
\gamma_{t}:=\#\left\{\sigma \in \operatorname{Hom}_{K}\left(L, K^{\mathrm{sep}}\right): i_{L / K}(\sigma) \geq t+1\right\}
$$

for $-1 \leq t \in \mathbb{R}$, and define the function $\varphi_{L / K}: \mathbb{R}_{\geq-1} \rightarrow \mathbb{R}_{\geq-1}$, the HasseHerbrand transition function of the extension $L / K$, by

$$
\varphi_{L / K}(u):= \begin{cases}\int^{u} \frac{\gamma_{t}}{\gamma_{0}} d t, & 0 \leq u \in \mathbb{R}, \\ u, & -1 \leq u \leq 0 .\end{cases}
$$

It is well-known that $\varphi_{L / K}: \mathbb{R}_{\geq-1} \rightarrow \mathbb{R}_{\geq-1}$ is a continuous, increasing, piecewise linear function, and it establishes a homeomorphism $\mathbb{R}_{\geq-1} \stackrel{\approx}{\rightarrow}$ $\mathbb{R}_{\geq-1}$. Let $\psi_{L / K}: \mathbb{R}_{\geq-1} \rightarrow \mathbb{R}_{\geq-1}$ be its inverse.

Assume that $L$ is a finite Galois extension over $K$ with Galois group $\operatorname{Gal}(L / K)=: G$. The normal subgroup $G_{u}$ of $G$ defined by

$$
G_{u}=\left\{\sigma \in G: i_{L / K}(\sigma) \geq u+1\right\}
$$

for $-1 \leq u \in \mathbb{R}$ is called the $u$ th ramification group of $G$ in the lower numbering, and has order $\gamma_{u}$. Note the inclusion $G_{u^{\prime}} \subseteq G_{u}$ for every pair $-1 \leq u, u^{\prime} \in \mathbb{R}$ satisfying $u \leq u^{\prime}$. The family $\left\{G_{u}\right\}_{u \in \mathbb{R} \geq-1}$ induces a filtration on $G$, called the lower ramification filtration of $G$. A break in this filtration is defined to be any number $u \in \mathbb{R}_{\geq-1}$ satisfying $G_{u} \neq G_{u+\varepsilon}$ for every $0<\varepsilon \in \mathbb{R}$. The function $\psi_{L / K}=\varphi_{L / K}^{-1}: \mathbb{R}_{\geq-1} \rightarrow \mathbb{R}_{\geq-1}$ induces the upper ramification filtration $\left\{G^{v}\right\}_{v \in \mathbb{R}_{\geq-1}}$ on $G$ by setting

$$
G^{v}:=G_{\psi_{L / K}(v)}
$$

or equivalently, by setting

$$
G^{\varphi_{L / K}(u)}=G_{u},
$$

for $-1 \leq v, u \in \mathbb{R}$; here $G^{v}$ is called the $v$ th upper ramification group of $G$. A break in the upper filtration $\left\{G^{v}\right\}_{v \in \mathbb{R}_{\geq-1}}$ of $G$ is defined to be any number $v \in \mathbb{R}_{\geq-1}$ satisfying $G^{v} \neq G^{v+\varepsilon}$ for every $0<\varepsilon \in \mathbb{R}$.

REMARK 2.1. We list the basic properties of lower and upper ramification filtrations on $G$. In what follows, $F / K$ denotes a sub-extension of $L / K$ and $H$ denotes the Galois group $\operatorname{Gal}(L / F)$. 
(i) The lower numbering on $G$ passes to the subgroup $H$ of $G$ in the sense that

$$
H_{u}=H \cap G_{u} \quad \text { for }-1 \leq u \in \mathbb{R} .
$$

(ii) If $H \triangleleft G$, then the upper numbering on $G$ passes to the quotient $G / H$ :

$$
(G / H)^{v}=G^{v} H / H \quad \text { for }-1 \leq v \in \mathbb{R} .
$$

(iii) The Hasse-Herbrand function and its inverse satisfy the transitive law

$$
\varphi_{L / K}=\varphi_{F / K} \circ \varphi_{L / F} \quad \text { and } \quad \psi_{L / K}=\psi_{L / F} \circ \psi_{F / K} .
$$

If $L / K$ is an infinite Galois extension with Galois group $\operatorname{Gal}(L / K)=G$, which is a topological group under the respective Krull topology, define the upper ramification filtration $\left\{G^{v}\right\}_{v \in \mathbb{R} \geq-1}$ on $G$ by the projective limit

$$
G^{v}:=\lim _{K \subseteq ્ \subseteq \subset} \operatorname{Gal}(F / K)^{v}
$$

over the transition morphisms $t_{F}^{F^{\prime}}(v): \operatorname{Gal}\left(F^{\prime} / K\right)^{v} \rightarrow \operatorname{Gal}(F / K)^{v}$, which are essentially the restriction morphisms from $F^{\prime}$ to $F$, defined naturally by the diagram

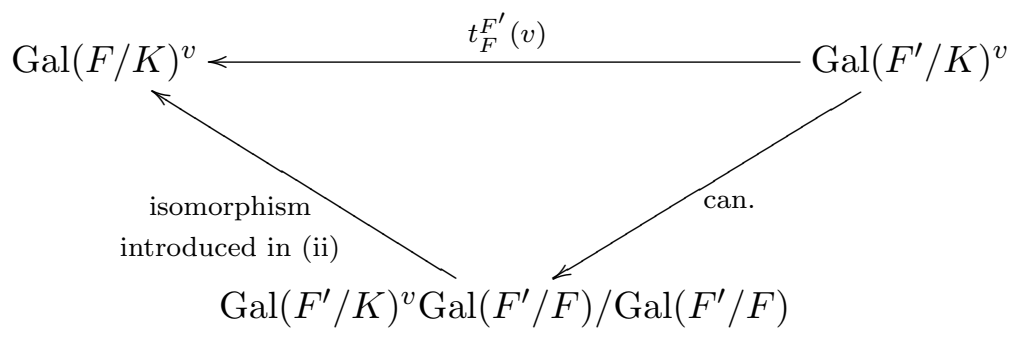

induced from (ii), as $K \subseteq F \subseteq F^{\prime} \subset L$ runs over all finite Galois extensions $F$ and $F^{\prime}$ over $K$ inside $L$. The topological subgroup $G^{v}$ of $G$ is called the $v$ th ramification group of $G$ in the upper numbering. Note the inclusion $G^{v^{\prime}} \subseteq G^{v}$ for every pair $-1 \leq v, v^{\prime} \in \mathbb{R}$ satisfying $v \leq v^{\prime}$ via the commutativity of the square

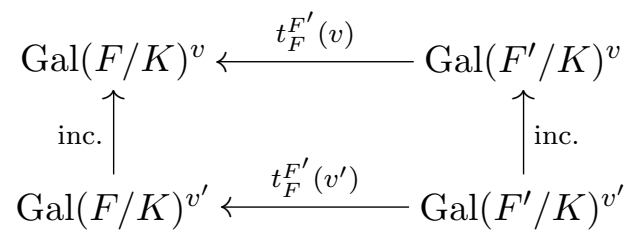

for every chain $K \subseteq F \subseteq F^{\prime} \subset L$ of finite Galois extensions $F$ and $F^{\prime}$ over $K$ inside $L$. Observe that:

(iv) $G^{-1}=G$ and $G^{0}$ is the inertia subgroup of $G$.

(v) $\bigcap_{v \in \mathbb{R} \geq-1} G^{v}=\left\langle 1_{G}\right\rangle$. 
(vi) $G^{v}$ is a closed subgroup of $G$, with respect to the Krull topology, for $-1 \leq v \in \mathbb{R}$.

In this setting, a number $-1 \leq v \in \mathbb{R}$ is said to be a break in the upper ramification filtration $\left\{G^{v}\right\}_{v \in \mathbb{R}>-1}$ of $G$, if $v$ is a break in the upper filtration of some finite quotient $G / H$ for some $H \triangleleft G$. Let $\mathcal{B}_{L / K}$ denote the set of all numbers $v \in \mathbb{R}_{\geq-1}$ which occur as breaks in the upper ramification filtration of $G$. Then:

(vii) (Hasse-Arf theorem) $\mathcal{B}_{K^{\mathrm{ab}} / K} \subseteq \mathbb{Z} \cap \mathbb{R}_{\geq-1}$.

(viii) $\mathcal{B}_{K^{\text {sep }} / K} \subseteq \mathbb{Q} \cap \mathbb{R}_{\geq-1}$.

2.4. APF-extensions. As in the previous section, let $\left\{G_{K}^{v}\right\}_{v \in \mathbb{R}_{\geq-1}}$ denote the upper ramification filtration of the absolute Galois group $G_{K}$ of $K$, and let $R^{v}$ denote the fixed field $\left(K^{\mathrm{sep}}\right)^{G_{K}^{v}}$ of the $v$ th upper ramification subgroup $G_{K}^{v}$ of $G_{K}$ in $K^{\text {sep }}$ for $-1 \leq v \in \mathbb{R}$.

Definition 2.2. An extension $L / K$ is called an APF-extension (APF is a shortening for "arithmétiquement profinie") if one of the following equivalent conditions is satisfied:

(i) $G_{K}^{v} G_{L}$ is open in $G_{K}$ for every $-1 \leq v \in \mathbb{R}$,

(ii) $\left(G_{K}: G_{K}^{v} G_{L}\right)<\infty$ for every $-1 \leq v \in \mathbb{R}$,

(iii) $L \cap R^{v}$ is a finite extension over $K$ for every $-1 \leq v \in \mathbb{R}$.

Note that if $L / K$ is an APF-extension, then $\left[\kappa_{L}: \kappa_{K}\right]<\infty$.

Now, let $L / K$ be an APF-extension. Set $G_{L}^{0}=G_{L} \cap G_{K}^{0}$, and define

$$
\psi_{L / K}(v)= \begin{cases}\int_{0}^{v}\left(G_{K}^{0}: G_{L}^{0} G_{K}^{x}\right) d x, & 0 \leq v \in \mathbb{R}, \\ v, & -1 \leq v \leq 0 .\end{cases}
$$

Then the map $v \mapsto \psi_{L / K}(v)$ for $v \in \mathbb{R}_{\geq-1}$, which is well-defined for the APF-extension $L / K$, defines a continuous, strictly increasing and piecewise linear bijection $\psi_{L / K}: \mathbb{R}_{\geq-1} \rightarrow \mathbb{R}_{\geq-1}$.

We denote the inverse of $\psi_{L / K}$ by $\varphi_{L / K}$. Thus, if $L / K$ is a (not necessarily finite) Galois APF-extension, then we can define the higher ramification subgroups in the lower numbering $\operatorname{Gal}(L / K)_{u}$ of $\operatorname{Gal}(L / K)$, for $-1 \leq u \in \mathbb{R}$, by setting

$$
\operatorname{Gal}(L / K)_{u}:=\operatorname{Gal}(L / K)^{\varphi_{L / K}(u)} .
$$

REMARK 2.3. Note that:

(i) In case $L / K$ is a finite separable extension, which is clearly an APFextension by Definition 2.2 , the function $\psi_{L / K}: \mathbb{R}_{\geq-1} \rightarrow \mathbb{R}_{\geq-1}$ coincides with the inverse of the Hasse-Herbrand transition function of $L / K$ introduced in the previous section. 
(ii) If $L / K$ is a finite separable extension and $L^{\prime} / L$ is an APF-extension, then $L^{\prime} / K$ is an APF-extension, and the transitivity rules for the functions $\psi_{L^{\prime} / K}, \varphi_{L^{\prime} / K}: \mathbb{R}_{\geq-1} \rightarrow \mathbb{R}_{\geq-1}$ hold:

$$
\psi_{L^{\prime} / K}=\psi_{L^{\prime} / L} \circ \psi_{L / K}, \quad \varphi_{L^{\prime} / K}=\varphi_{L / K} \circ \varphi_{L^{\prime} / L} .
$$

3. Non-abelian local reciprocity map. In this section, we shall review the theory developed in [Ik-Se-2, Ik-Se-3]. Fix a Lubin-Tate splitting $\varphi$ over $K$.

3.1. Generalized Fesenko reciprocity map. For an infinite APFGalois extension $L / K$ with residue class degree $\left[\kappa_{L}: \kappa_{K}\right]=d$ and with $K \subset$ $L \subset K_{\varphi^{d}}$, denote the field of norms corresponding to $L / K$ by $\mathbb{X}(L / K)$ and the completion of the maximal unramified extension $\mathbb{X}(L / K)^{\mathrm{nr}}$ of $\mathbb{X}(L / K)$ by $\widetilde{\mathbb{X}}(L / K)$ (for details, [Fe-Vo, [Fo-Wi-1, Fo-Wi-2] and [Win]), and set $L_{0}=L \cap K^{\mathrm{nr}}$. There exists a bijective 1-cocycle

$$
\boldsymbol{\Phi}_{L / K}^{(\varphi)}: \operatorname{Gal}(L / K) \rightarrow K^{\times} / N_{L_{0} / K} L_{0}^{\times} \times U_{\widetilde{\mathbb{X}}(L / K)}^{\diamond} / Y_{L / L_{0}},
$$

called the generalized Fesenko reciprocity map for the extension $L / K$, defined by the composition

$$
\begin{aligned}
& \operatorname{Gal}(L / K) \stackrel{\phi_{L / K}^{(\varphi)}}{\longrightarrow} K^{\times} / N_{L_{0} / K} L_{0}^{\times} \times U_{\widetilde{\mathbb{X}}(L / K)}^{\diamond} / U_{\mathbb{X}(L / K)}
\end{aligned}
$$

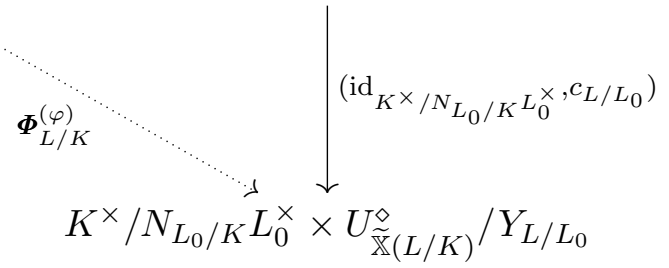

Here,

$$
\phi_{L / K}^{(\varphi)}: \operatorname{Gal}(L / K) \rightarrow K^{\times} / N_{L_{0} / K} L_{0}^{\times} \times U_{\widetilde{\mathbb{X}}(L / K)}^{\diamond} / U_{\mathbb{X}(L / K)}
$$

is an injective 1-cocycle called, following [Ik-Se-2, the generalized arrow defined for the extension $L / K$, and defined by

$$
\phi_{L / K}^{(\varphi)}(\sigma)=\left(\pi_{K}^{m} N_{L_{0} / K} L_{0}^{\times}, \phi_{L / L_{0}}^{\left(\varphi^{d}\right)}\left(\varphi^{-m} \sigma\right)\right)
$$

for every $\sigma \in \operatorname{Gal}(L / K)$, where $0 \leq m \in \mathbb{Z}$ is the integer satisfying $\left.\sigma\right|_{L_{0}}=$ $\left.\varphi^{m}\right|_{L_{0}} \in \operatorname{Gal}\left(L_{0} / K\right)$ and $\varphi^{-m} \sigma \in \operatorname{Gal}\left(L / L_{0}\right)$, and for any $\tau \in \operatorname{Gal}\left(L / L_{0}\right)$, the value $\phi_{L / L_{0}}^{\left(\varphi^{d}\right)}(\tau)$ of the arrow defined for the extension $L / L_{0}$ at $\tau$ is defined by [Fes-1, Fes-2, Fes-3] and [1k-Se-1]. Namely, $\phi_{L / L_{0}}^{\left(\varphi^{d}\right)}(\tau)=U_{\tau} \cdot U_{\mathbb{X}\left(L / L_{0}\right)}$ provided that $U_{\tau} \in U_{\widetilde{\mathbb{X}}(L / K)}^{\diamond}$, which is unique modulo $U_{\mathbb{X}\left(L / L_{0}\right)}$, solves the equation $U^{1-\varphi^{d}}=\Pi_{\varphi^{d} ; L / L_{0}}^{\tau-1}$, where $\Pi_{\varphi^{d} ; L / L_{0}}$ is the canonical prime element 
of the local field $\mathbb{X}\left(L / L_{0}\right)$ defined in Lemmas 1.2 and 1.3 of [Ik-Se-2]. For the definition of the group $U_{\widetilde{\mathbb{X}}(L / K)}^{\diamond}$ and its subgroups $U_{\mathbb{X}\left(L / L_{0}\right)}$ and $Y_{L / L_{0}}$ satisfying the inclusion $U_{\mathbb{X}\left(L / L_{0}\right)} \subseteq Y_{L / L_{0}}$ we refer the reader to [Fes-1, Fes-2, Fes-3] and [k-Se-1]. In the commutative triangle (3.2), the arrow

$$
c_{L / L_{0}}: U_{\widetilde{\mathbb{X}}(L / K)}^{\diamond} / U_{\mathbb{X}(L / K)} \rightarrow U_{\widetilde{\mathbb{X}}(L / K)}^{\diamond} / Y_{L / L_{0}}
$$

is the canonical map defined by the inclusion $U_{\mathbb{X}\left(L / L_{0}\right)} \subseteq Y_{L / L_{0}}$. Recall that (cf. [Fes-1, Fes-2, Fes-3] and [Ik-Se-1]) the composition $c_{L / L_{0}} \circ \phi_{L / L_{0}}^{\left(\varphi^{d}\right)}=$ $\Phi_{L / L_{0}}^{\left(\varphi^{d}\right)}: \operatorname{Gal}\left(L / L_{0}\right) \rightarrow U_{\widetilde{\mathbb{X}}(L / K)}^{\diamond} / Y_{L / L_{0}}$ is the Fesenko reciprocity map for the extension $L / L_{0}$. Thus, for $\sigma \in \operatorname{Gal}(L / K)$, the value $\boldsymbol{\Phi}_{L / K}^{(\varphi)}(\sigma)$ is defined by

$$
\boldsymbol{\Phi}_{L / K}^{(\varphi)}(\sigma)=\left(\pi_{K}^{m} N_{L_{0} / K} L_{0}^{\times}, \Phi_{L / L_{0}}^{\left(\varphi^{d}\right)}\left(\varphi^{-m} \sigma\right)\right),
$$

where $0 \leq m \in \mathbb{Z}$ satisfies $\left.\sigma\right|_{L_{0}}=\left.\varphi^{m}\right|_{L_{0}} \in \operatorname{Gal}\left(L_{0} / K\right)$ and $\varphi^{-m} \sigma \in$ $\operatorname{Gal}\left(L / L_{0}\right)$.

Define a composition law $*$ on $\operatorname{im}\left(\phi_{L / K}^{(\varphi)}\right)$ by

$$
(\bar{a}, \bar{U}) *(\bar{b}, \bar{V})=(\bar{a}, \bar{U}) \cdot(\bar{b}, \bar{V})^{\left(\phi_{L / K}^{(\varphi)}\right)^{-1}((\bar{a}, \bar{U}))}
$$

for every $\bar{a}=a \cdot N_{L_{0} / K} L_{0}^{\times}, \bar{b}=b . N_{L_{0} / K} L_{0}^{\times} \in K^{\times} / N_{L_{0} / K} L_{0}^{\times}$with $a, b \in$ $K^{\times}$and $\bar{U}=U \cdot U_{\mathbb{X}(L / K)}, \bar{V}=V \cdot U_{\mathbb{X}(L / K)} \in U_{\widetilde{\mathbb{X}}(L / K)}^{\diamond} / U_{\mathbb{X}(L / K)}$ with $U, V \in$ $U_{\widetilde{\mathbb{X}}(L / K)}^{\diamond}$, where the action of $\operatorname{Gal}(L / K)$ on $\operatorname{im}\left(\phi_{L / K}^{(\varphi)}\right)$ is defined by $(\bar{b}, \bar{V})^{\sigma}=$ $\left(\bar{b}, \bar{V}^{\varphi^{-m} \sigma}\right)$. Then $K^{\times} / N_{L_{0} / K} L_{0}^{\times} \times U_{\widetilde{\mathbb{X}}(L / K)}^{\otimes} / U_{\mathbb{X}(L / K)}$ is a topological group under $*$, and $\boldsymbol{\phi}_{L / K}^{(\varphi)}$ induces an isomorphism of topological groups

$$
\phi_{L / K}^{(\varphi)}: \operatorname{Gal}(L / K) \stackrel{\sim}{\longrightarrow} \operatorname{im}\left(\phi_{L / K}^{(\varphi)}\right),
$$

where the topological group structure on $\operatorname{im}\left(\phi_{L / K}^{(\varphi)}\right)$ is defined with respect to the binary operation $*$ defined by (3.7). Likewise, define a composition law, again denoted by $*$, on $K^{\times} / N_{L_{0} / K} L_{0}^{\times} \times U_{\widetilde{\mathbb{X}}(L / K)}^{\diamond} / Y_{L / L_{0}}$ by

$$
(\bar{a}, \bar{U}) *(\bar{b}, \bar{V})=(\bar{a}, \bar{U}) \cdot(\bar{b}, \bar{V})^{\left(\Phi_{L / K}^{(\varphi)}\right)^{-1}((\bar{a}, \bar{U}))}
$$

for every $\bar{a}=a \cdot N_{L_{0} / K} L_{0}^{\times}, \bar{b}=b \cdot N_{L_{0} / K} L_{0}^{\times} \in K^{\times} / N_{L_{0} / K} L_{0}^{\times}$with $a, b \in K^{\times}$ and $\bar{U}=U \cdot Y_{L / L_{0}}, \bar{V}=V \cdot Y_{L / L_{0}} \in U_{\widetilde{\mathbb{X}}(L / K)}^{\diamond} / Y_{L / L_{0}}$ with $U, V \in U_{\widetilde{\mathbb{X}}(L / K)}^{\diamond}$, where the action of $\operatorname{Gal}(L / K)$ on $K^{\times} / N_{L_{0} / K} L_{0}^{\times} \times U_{\widetilde{\mathbb{X}}(L / K)}^{\diamond} / Y_{L / L_{0}}$ is defined by $(\bar{b}, \bar{V})^{\sigma}=\left(\bar{b}, \bar{V}^{\varphi^{-m} \sigma}\right)$. Then $K^{\times} / N_{L_{0} / K} L_{0}^{\times} \times U_{\widetilde{\mathbb{X}}(L / K)}^{\diamond} / Y_{L / L_{0}}$ is a topolog- 
ical group under $*$, and $\boldsymbol{\Phi}_{L / K}^{(\varphi)}$ induces an isomorphism of topological groups

$$
\boldsymbol{\Phi}_{L / K}^{(\varphi)}: \operatorname{Gal}(L / K) \stackrel{\sim}{\rightarrow} K^{\times} / N_{L_{0} / K} L_{0}^{\times} \times U_{\widetilde{\mathbb{X}}(L / K)}^{\diamond} / Y_{L / L_{0}},
$$

where the topological group structure on $K^{\times} / N_{L_{0} / K} L_{0}^{\times} \times U_{\widetilde{\mathbb{X}}(L / K)}^{\diamond} / Y_{L / L_{0}}$ is defined with respect to the binary operation $*$ defined by $(3.9)$.

The mappings $\phi_{L / K}^{(\varphi)}$ and $\boldsymbol{\Phi}_{L / K}^{(\varphi)}$ have the following basic properties.

(i) For an infinite Galois sub-extension $M / K$ of $L / K$ such that $\left[\kappa_{M}: \kappa_{K}\right]$ $=d^{\prime}$ and $K \subset M \subset K_{\varphi^{d^{\prime}}}$ for some $d^{\prime} \mid d$, the square

$$
\begin{aligned}
& \operatorname{Gal}(L / K) \stackrel{\phi_{L / K}^{(\varphi)}}{\longrightarrow} K^{\times} / N_{L_{0} / K} L_{0}^{\times} \times U_{\widetilde{\mathbb{X}}(L / K)}^{\diamond} / U_{\mathbb{X}(L / K)}
\end{aligned}
$$

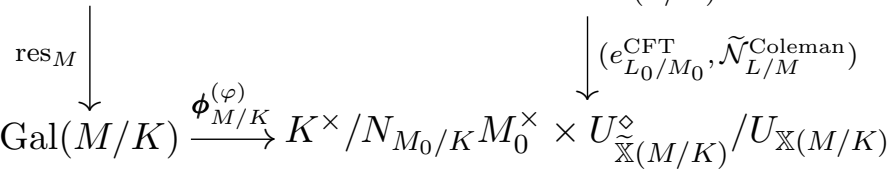

is commutative, where the right vertical arrow is defined by

$$
\left(e_{L_{0} / M_{0}}^{\mathrm{CFT}}, \tilde{\mathcal{N}}_{L / M}^{\text {Coleman }}\right):(\bar{a}, \bar{U}) \mapsto\left(e_{L_{0} / M_{0}}^{\mathrm{CFT}}(\bar{a}), \tilde{\mathcal{N}}_{L / M}^{\text {Coleman }}(\bar{U})\right)
$$

for every $(\bar{a}, \bar{U}) \in K^{\times} / N_{L_{0} / K} L_{0}^{\times} \times U_{\widetilde{\mathbb{X}}(L / K)}^{\diamond} / U_{\mathbb{X}(L / K)}$. Here,

$$
\tilde{\mathcal{N}}_{L / M}^{\text {Coleman }}: U_{\mathbb{\mathbb { X }}(L / K)}^{\diamond} / U_{\mathbb{X}(L / K)} \rightarrow U_{\widetilde{\mathbb{X}}(M / K)}^{\diamond} / U_{\mathbb{X}(M / K)}
$$

is the Coleman norm map from $L$ to $M$ defined by equations (2.22) and (2.23) of [Ik-Se-2]. Likewise, the square

$$
\begin{aligned}
\operatorname{Gal}(L / K) \stackrel{\Phi_{L / K}^{(\varphi)}}{\longrightarrow} K^{\times} / N_{L_{0} / K} L_{0}^{\times} \times U_{\widetilde{\mathbb{X}}(L / K)}^{\diamond} / Y_{L / L_{0}} \\
\underset{\operatorname{res}_{M} \downarrow}{\downarrow} \underset{\left(e_{L_{0} / M_{0}}^{\mathrm{CFT}}, \widetilde{\mathcal{N}}_{L / M}^{\text {Coleman }}\right)}{\operatorname{Gal}(M / K) \stackrel{\boldsymbol{\Phi}_{M / K}^{(\varphi)}}{\longrightarrow} K^{\times} / N_{M_{0} / K} M_{0}^{\times} \times U_{\widetilde{\mathbb{X}}(M / K)}^{\ominus} / Y_{M / M_{0}}}
\end{aligned}
$$

is commutative, where the right vertical arrow is defined by

$$
\left(e_{L_{0} / M_{0}}^{\mathrm{CFT}}, \tilde{\mathcal{N}}_{L / M}^{\text {Coleman }}\right):(\bar{a}, \bar{U}) \mapsto\left(e_{L_{0} / M_{0}}^{\mathrm{CFT}}(\bar{a}), \tilde{\mathcal{N}}_{L / M}^{\text {Coleman }}(\bar{U})\right)
$$

for $(\bar{a}, \bar{U}) \in K^{\times} / N_{L_{0} / K} L_{0}^{\times} \times U_{\widetilde{\mathbb{X}}(L / K)}^{\diamond} / Y_{L / L_{0}}$. Here, $\tilde{\mathcal{N}}_{L / M}^{\text {Coleman }}: U_{\widetilde{\mathbb{X}}(L / K)}^{\diamond} / Y_{L / L_{0}}$ $\rightarrow U_{\widetilde{\mathbb{X}}(M / K)}^{\diamond} / Y_{M / M_{0}}$ is the Coleman norm map from $L$ to $M$ defined by Lemma 2.21 together with equations (2.47) and (2.48) of [Ik-Se-2]. Moreover, the arrow $e_{L_{0} / M_{0}}^{\mathrm{CFT}}: K^{\times} / N_{L_{0} / K} L_{0}^{\times} \rightarrow K^{\times} / N_{M_{0} / K} M_{0}^{\times}$appearing in both commutative diagrams is the natural inclusion defined via the existence theorem of local class field theory. 
(ii) For each $0 \leq i \in \mathbb{R}$, introduce the subgroups $\left(U_{\widetilde{\mathbb{X}}(L / K)}^{\diamond}\right)^{i}$ of the field $\widetilde{\mathbb{X}}(L / K)$ by $\left(U_{\widetilde{\mathbb{X}}(L / K)}^{\diamond}\right)^{i}=U_{\widetilde{\mathbb{X}}(L / K)}^{\diamond} \cap U_{\widetilde{\mathbb{X}}(L / K)}^{i}$. For each $0 \leq n \in \mathbb{Z}$, as in equation (5.42) of [Ik-Se-1], let

$$
Q_{L / L_{0}}^{n}=c_{L / L_{0}}\left(\left(U_{\widetilde{\mathbb{X}}(L / K)}^{\diamond}\right)^{n} U_{\mathbb{X}(L / K)} / U_{\mathbb{X}(L / K)} \cap \operatorname{im}\left(\phi_{L / L_{0}}^{\left(\varphi^{d}\right)}\right)\right),
$$

which is a subgroup of $\left(U_{\widetilde{\mathbb{X}}(L / K)}^{\diamond}\right)^{n} Y_{L / L_{0}} / Y_{L / L_{0}}$. Here, the canonical homomorphism $c_{L / L_{0}}$ introduced in (3.5) is defined by equation (5.35) of [Ik-Se-1]. Now, the ramification theorem for the generalized arrow $\phi_{L / K}^{(\varphi)}$ yields, for $0 \leq n \in \mathbb{Z}$, the inclusion

$$
\begin{gathered}
\phi_{L / K}^{(\varphi)}\left(\operatorname{Gal}(L / K)_{\psi_{L / K} \circ \varphi_{L / L_{0}}(n)}-\operatorname{Gal}(L / K)_{\psi_{L / K^{\circ}} \circ \varphi_{L / L_{0}}(n+1)}\right) \\
\subseteq\left\langle 1_{K^{\times} / N_{L_{0} / K} L_{0}^{\times}}\right\rangle \times\left(\left(U_{\widetilde{\mathbb{X}}(L / K)}^{\diamond}\right)^{n} U_{\mathbb{X}(L / K)} / U_{\mathbb{X}(L / K)}\right. \\
\left.-\left(U_{\widetilde{\mathbb{X}}(L / K)}^{\diamond}\right)^{n+1} U_{\mathbb{X}(L / K)} / U_{\mathbb{X}(L / K)}\right),
\end{gathered}
$$

and the ramification theorem for the generalized Fesenko reciprocity map $\boldsymbol{\Phi}_{L / K}^{(\varphi)}$ gives, for $0 \leq n \in \mathbb{Z}$, the inclusion

$$
\begin{aligned}
\boldsymbol{\Phi}_{L / K}^{(\varphi)}\left(\operatorname{Gal}(L / K)_{\psi_{L / K} \circ \varphi_{L / L_{0}}}(n)\right. & \left.-\operatorname{Gal}(L / K)_{\psi_{L / K} \circ \varphi_{L / L_{0}}}(n+1)\right) \\
\subseteq & \left(1_{K^{\times} / N_{L_{0} / K} L_{0}^{\times}}\right\rangle \times\left(\left(U_{\widetilde{\mathbb{X}}(L / K)}^{\ominus}\right)^{n} Y_{L / L_{0}} / Y_{L / L_{0}}-Q_{L / L_{0}}^{n+1}\right),
\end{aligned}
$$

where, for $0 \leq u \in \mathbb{R}, \operatorname{Gal}(L / K)_{u}$ denotes the $u$ th ramification subgroup in the lower numbering of the Galois group $\operatorname{Gal}(L / K)$ corresponding to the infinite APF-Galois extension $L / K$.

REMARK 3.1. In fact, ramification theorems for $\phi_{L / K}^{(\varphi)}$ and $\boldsymbol{\Phi}_{L / K}^{(\varphi)}$ stated in (3.16) and (3.17) can be simplified as follows. For $0 \leq n \in \mathbb{Z}$, as $\varphi_{L / K}(n)=$ $\varphi_{L_{0} / K} \circ \varphi_{L / L_{0}}(n)$ and $L_{0}=L \cap K^{\mathrm{nr}}$, it follows that $\varphi_{L / K}(n)=\varphi_{L / L_{0}}(n)$. Therefore, (3.16) can be reformulated as

$$
\begin{aligned}
& \boldsymbol{\phi}_{L / K}^{(\varphi)}\left(\operatorname{Gal}(L / K)_{n}-\operatorname{Gal}(L / K)_{n+1}\right) \subseteq\left\langle 1_{K^{\times} / N_{L_{0} / K} L_{0}^{\times}}\right\rangle \\
& \times\left(\left(U_{\widetilde{\mathbb{X}}(L / K)}^{\diamond}\right)^{n} U_{\mathbb{X}(L / K)} / U_{\mathbb{X}(L / K)}-\left(U_{\widetilde{\mathbb{X}}(L / K)}^{\diamond}\right)^{n+1} U_{\mathbb{X}(L / K)} / U_{\mathbb{X}(L / K)}\right),
\end{aligned}
$$

and (3.17) can be reformulated as

$$
\begin{aligned}
\boldsymbol{\Phi}_{L / K}^{(\varphi)}( & \left.\operatorname{Gal}(L / K)_{n}-\operatorname{Gal}(L / K)_{n+1}\right) \\
& \subseteq\left\langle 1_{K^{\times} / N_{L_{0} / K} L_{0}^{\times}}\right\rangle \times\left(\left(U_{\widetilde{\mathbb{X}}(L / K)}^{\ominus}\right)^{n} Y_{L / L_{0}} / Y_{L / L_{0}}-Q_{L / L_{0}}^{n+1}\right) .
\end{aligned}
$$

Finally, the following remark is in order.

REMARK 3.2. We do not need assumption (2.1) on the local field $K$ to define the generalized arrow $\phi_{L / K}^{(\varphi)}$ by (3.4). For details, cf. [Ik-Se-2]. 
3.2. Construction of the non-abelian local reciprocity map. For each $1 \leq d \in \mathbb{Z}$, let $K_{\varphi^{d}}$ denote the fixed field of $\varphi^{d} \in G_{K}$. Observe that $K^{\mathrm{sep}}=K^{\mathrm{nr}} K_{\varphi^{d}}$ and $K_{d}^{\mathrm{nr}}=K^{\mathrm{nr}} \cap K_{\varphi^{d}}$, where $K_{d}^{\mathrm{nr}}$ denotes the unique unramified extension over $K$ of degree $d$. Now, for each $1 \leq n, d \in \mathbb{Z}$, let $\Gamma_{d}^{(n)}:=\Gamma_{d}^{(n)}(K, \varphi)$ be a Galois extension over $K$, which is the unique maximal $n$-abelian extension $\left({ }^{1}\right)$ of $K_{d}^{\mathrm{nr}}$ in $K_{\varphi^{d}}$. Note that

$$
\bigcup_{1 \leq d \in \mathbb{Z}} \Gamma_{d}^{(n)}=\left(K^{\mathrm{nr}}\right)^{n-\mathrm{ab}},
$$

where $\left(K^{\mathrm{nr}}\right)^{n \text {-ab }}$ denotes the " $n$-abelian closure" of $K^{\mathrm{nr}}$ in $K^{\text {sep }}$. Thus, it also follows that

$$
\bigcup_{1 \leq n \in \mathbb{Z}} \bigcup_{1 \leq d \in \mathbb{Z}} \Gamma_{d}^{(n)}=K^{\text {sep }}
$$

Moreover, for each pair $(n, d)$ of positive integers, $\Gamma_{d}^{(n)}$ is an APF-extension over $K$.

Now, the absolute Galois group $G_{K}$ of the local field $K$ is the projective limit

$$
G_{K}=\lim _{(n, d)} \operatorname{Gal}\left(\Gamma_{d}^{(n)} / K\right)
$$

over the restriction morphisms

$$
r_{\left(n^{\prime}, d^{\prime}\right)}^{(n, d)}: \operatorname{Gal}\left(\Gamma_{d}^{(n)} / K\right) \rightarrow \operatorname{Gal}\left(\Gamma_{d^{\prime}}^{\left(n^{\prime}\right)} / K\right)
$$

for $(n, d),\left(n^{\prime}, d^{\prime}\right) \in \mathbb{Z}_{\geq 1} \times \mathbb{Z}_{\geq 1}$ satisfying $n^{\prime} \leq n$ and $d^{\prime} \mid d$ (which is equivalent to $\left.\Gamma_{d^{\prime}}^{\left(n^{\prime}\right)} \subseteq \Gamma_{d}^{(n)}\right)$. Note that, for each $1 \leq n, d \in \mathbb{Z}$, the APF-Galois extension $\Gamma_{d}^{(n)}$ over $K$ has the residue class degree $d$. Therefore, the generalized Fesenko theory developed in [Ik-Se-2] can be applied to the extensions of the form $\Gamma_{d}^{(n)} / K$, which would enable us to construct the generalized arrow $\phi_{\Gamma_{d}^{(n)} / K}^{(\varphi)}$ and the generalized Fesenko reciprocity map $\Phi_{\Gamma_{d}^{(n)} / K}^{(\varphi)}$, for every pair $(n, d) \in \mathbb{Z}_{\geq 1} \times \mathbb{Z}_{\geq 1}$. Then using property (i) for the collections $\left\{\boldsymbol{\phi}_{\Gamma_{d}^{(n)} / K}^{(\varphi)}\right\}_{(n, d) \in \mathbb{Z}_{\geq 1} \times \mathbb{Z}_{\geq 1}}$ and $\left\{\boldsymbol{\Phi}_{\Gamma_{d}^{(n)} / K}^{(\varphi)}\right\}_{(n, d) \in \mathbb{Z}_{\geq 1} \times \mathbb{Z}_{\geq 1}}$, and passing to the projective limits, we get the generalized arrow $\boldsymbol{\phi}_{K}^{(\varphi)}$ for the local field $K$ and the non-abelian local reciprocity map $\boldsymbol{\Phi}_{K}^{(\varphi)}$ for the local field $K$ respectively.

To be more precise, we first introduce the following notation to simplify the discussion. In what follows, $L / K$ denotes an infinite APF-Galois extension such that $\left[\kappa_{L}: \kappa_{K}\right]=d$ and $K \subset L \subset K_{\varphi^{d}}$.

$\left({ }^{1}\right)$ Recall that by an $n$-abelian extension over a field $F$, we mean a Galois extension $E / F$ whose Galois group $\operatorname{Gal}(E / F)$ has a trivial $n$th commutator subgroup $\operatorname{Gal}(E / F)^{(n)}$. 
Notation 3.3. For an infinite Galois sub-extension $M / K$ of $L / K$ such that $\left[\kappa_{M}: \kappa_{K}\right]=d^{\prime}$ and $K \subset M \subset K_{\varphi^{d^{\prime}}}$ provided that $d^{\prime} \mid d$, we let

(i) $\mathcal{C}_{L / M}^{o}$ denote the map $\left(e_{L_{0} / M_{0}}^{\mathrm{CFT}}, \tilde{\mathcal{N}}_{L / M}^{\text {Coleman }}\right)$ defined by (3.11) and (3.12),

(ii) $\mathcal{C}_{L / M}$ denote the map $\left(e_{L_{0} / M_{0}}^{\mathrm{CFT}}, \tilde{\mathcal{N}}_{L / M}^{\text {Coleman }}\right)$ defined by (3.13) and (3.14).

Recall that

$$
\begin{aligned}
\mathcal{C}_{L / M}^{o}: K^{\times} / N_{L_{0} / K} L_{0}^{\times} \times U_{\widetilde{\mathbb{X}}(L / K)}^{\diamond} / & U_{\mathbb{X}(L / K)} \\
& \rightarrow K^{\times} / N_{M_{0} / K} M_{0}^{\times} \times U_{\widetilde{\mathbb{X}}(M / K)}^{\ominus} / U_{\mathbb{X}(M / K)}
\end{aligned}
$$

and

$\mathcal{C}_{L / M}: K^{\times} / N_{L_{0} / K} L_{0}^{\times} \times U_{\widetilde{\mathbb{X}}(L / K)}^{\diamond} / Y_{L / L_{0}} \rightarrow K^{\times} / N_{M_{0} / K} M_{0}^{\times} \times U_{\widetilde{\mathbb{X}}(M / K)}^{\diamond} / Y_{M / M_{0}}$ are homomorphisms of the underlying abelian groups. Moreover, for the valued fields $L$ and $M$ as above, let $F / K$ be an infinite Galois sub-extension of $M / K$ satisfying $K \subset F \subset K_{\varphi d^{\prime \prime}}$ with $\left[\kappa_{F}: \kappa_{K}\right]=d^{\prime \prime}$ where $d^{\prime \prime} \mid d^{\prime}$. If we set $F_{0}=F \cap K^{\mathrm{nr}}$, the following equalities hold:

(i) $\mathcal{C}_{L / M}^{o}=$ id and $\mathcal{C}_{L / M}=$ id, if $L=M$.

(ii) $\mathcal{C}_{L / F}^{o}=\mathcal{C}_{M / F}^{o} \circ \mathcal{C}_{L / M}^{o}$ and $\mathcal{C}_{L / F}=\mathcal{C}_{M / F} \circ \mathcal{C}_{L / M}$.

It follows that the systems

$$
\left\{K^{\times} / N_{K_{d}^{\mathrm{nr}} / K} K_{d}^{\mathrm{nr} \times} \times U_{\widetilde{\mathbb{X}}\left(\Gamma_{d}^{(n)} / K\right)}^{\diamond} / U_{\mathbb{X}\left(\Gamma_{d}^{(n)} / K\right)} ; \mathcal{C}_{\Gamma_{d}^{(n)} / \Gamma_{d^{\prime}}^{\left(n^{\prime}\right)}}^{\infty}\right\}_{n^{\prime} \leq n} d_{d^{\prime} \mid d}
$$

and

$$
\left\{K^{\times} / N_{K_{d}^{\mathrm{nr}} / K} K_{d}^{\mathrm{nr} \times} \times U_{\widetilde{\mathbb{X}}\left(\Gamma_{d}^{(n)} / K\right)}^{\diamond} / Y_{\Gamma_{d}^{(n)} / K_{d}^{\mathrm{nr}}} ; \mathcal{C}_{\Gamma_{d}^{(n)} / \Gamma_{d^{\prime}}^{\left(n^{\prime}\right)}}\right\}_{n^{\prime} \leq n} d_{d^{\prime} \mid d}
$$

are projective. Let

$$
\begin{aligned}
& \nabla_{K}^{(\varphi), o}=\nabla_{K}^{o}=\lim _{(n, d)} K^{\times} / N_{K_{d}^{\mathrm{nr}} / K} K_{d}^{\mathrm{nr} \times} \times U_{\widetilde{\mathbb{X}}\left(\Gamma_{d}^{(n)} / K\right)} / U_{\mathbb{X}\left(\Gamma_{d}^{(n)} / K\right)} \\
& =\widehat{\mathbb{Z}} \times{\underset{(i m}{(n, d)}}_{\lim _{\widetilde{\mathbb{X}}\left(\Gamma_{d}^{(n)} / K\right)}} / U_{\mathbb{X}\left(\Gamma_{d}^{(n)} / K\right)}
\end{aligned}
$$

and

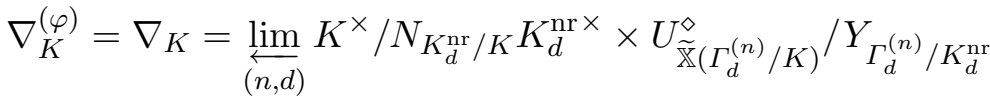

$$
\begin{aligned}
& =\widehat{\mathbb{Z}} \times \lim _{(n, d)} U_{\widetilde{\mathbb{X}}\left(\Gamma_{d}^{(n)} / K\right)}^{\diamond} / Y_{\Gamma_{d}^{(n)} / K_{d}^{\mathrm{nr}}}
\end{aligned}
$$

be the projective limits of the systems $(3.22)$ and $(3.23)$ respectively. The limits $\nabla_{K}^{(\varphi), o}$ and $\nabla_{K}^{(\varphi)}$, or $\nabla_{K}^{o}$ and $\nabla_{K}$ respectively if there is no risk of confusion, depend on the choice of a Lubin-Tate splitting $\varphi$ over $K$. 
Note that $\nabla_{K}^{o}$ and $\nabla_{K}$ have natural topological $G_{K}$-module structures, where the $G_{K}$-action on $\nabla_{K}^{o}$ and on $\nabla_{K}$ is defined by

$$
\left(\left(\bar{a}_{d, n}, \bar{U}_{d, n}\right)\right)_{d, n}^{\sigma}=\left(\left(\bar{a}_{d, n}, \bar{U}_{d, n}\right)^{\left.\sigma\right|_{d} ^{(n)}}\right)_{d, n}
$$

for every coherent sequence $\left(\left(\bar{a}_{d, n}, \bar{U}_{d, n}\right)\right)_{d, n}$ from $\nabla_{K}^{o}$ or from $\nabla_{K}$, and for every $\sigma \in G_{K}=\lim _{(n, d)} \operatorname{Gal}\left(\Gamma_{d}^{(n)} / K\right)$

For any two pairs $(n, d)$ and $\left(n^{\prime}, d^{\prime}\right)$ satisfying $n^{\prime} \leq n$ and $d^{\prime} \mid d$, the square

$$
\begin{aligned}
& U_{\widetilde{\mathbb{X}}\left(\Gamma_{d}^{(n)} / K\right)} / U_{\mathbb{X}\left(\Gamma_{d}^{(n)} / K\right)} \stackrel{c_{\Gamma_{d}^{(n)} / K_{d}^{\mathrm{nr}}}^{\longrightarrow}}{\longrightarrow} U_{\widetilde{\mathbb{X}}\left(\Gamma_{d}^{(n)} / K\right)} / Y_{\Gamma_{d}^{(n)} / K_{d}^{\mathrm{nr}}}
\end{aligned}
$$

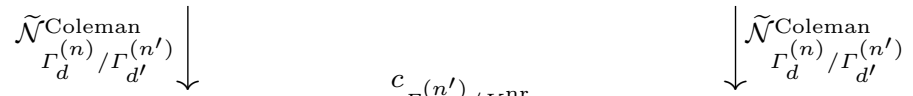

$$
\begin{aligned}
& U_{\widetilde{\mathbb{X}}\left(\Gamma_{d^{\prime}}^{\left(n^{\prime}\right)} / K\right)}^{\diamond} / U_{\mathbb{X}\left(\Gamma_{d^{\prime}}^{\left(n^{\prime}\right)} / K\right) \stackrel{\Gamma_{d^{\prime}}^{\left(n^{\prime}\right)} / K_{d^{\prime}}^{\mathrm{nr}}}{\longrightarrow}} U_{\widetilde{\mathbb{X}}\left(\Gamma_{d^{\prime}}^{\left(n^{\prime}\right)} / K\right)} / Y_{\Gamma_{d^{\prime}}^{\left(n^{\prime}\right)} / K_{d^{\prime}}^{\mathrm{nr}}}
\end{aligned}
$$

is commutative. Therefore, the topological $G_{K}$-modules $\nabla_{K}^{o}$ and $\nabla_{K}$ are related to each other by a topological $G_{K}$-module homomorphism

$$
c_{K}:=\lim _{(n, d)}\left(\operatorname{id}_{K \times} / N_{K_{d}^{\mathrm{nr}} / K} K_{d}^{\mathrm{nr} \times}, c_{\Gamma_{d}^{(n)} / K_{d}^{\mathrm{nr}}}\right): \nabla_{K}^{o} \rightarrow \nabla_{K}
$$

defined by the commutativity of the diagram (3.27).

Therefore, there exists an injective map

$$
\phi_{K}^{(\varphi)}=\lim _{(n, d)} \phi_{\Gamma_{d}^{(n)} / K}^{(\varphi)}: G_{K} \rightarrow \nabla_{K}^{o}
$$

defined by

$$
\boldsymbol{\phi}_{K}^{(\varphi)}\left(\left(\sigma_{d, n}\right)_{d, n}\right)=\left(\boldsymbol{\phi}_{\Gamma_{d}^{(n)} / K}^{(\varphi)}\left(\sigma_{d, n}\right)\right)_{d, n}
$$

for every coherent sequence $\left(\sigma_{d, n}\right)_{d, n} \in \underset{(n, d)}{\lim } \operatorname{Gal}\left(\Gamma_{d}^{(n)} / K\right)=G_{K}$, and a bi-
jective map

$$
\boldsymbol{\Phi}_{K}^{(\varphi)}=\lim _{(n, d)} \boldsymbol{\Phi}_{\Gamma_{d}^{(n)} / K}^{(\varphi)}: G_{K} \rightarrow \nabla_{K}
$$

defined by

$$
\boldsymbol{\Phi}_{K}^{(\varphi)}\left(\left(\sigma_{d, n}\right)_{d, n}\right)=\left(\boldsymbol{\Phi}_{\Gamma_{d}^{(n)} / K}^{(\varphi)}\left(\sigma_{d, n}\right)\right)_{d, n}
$$

for every coherent sequence $\left(\sigma_{d, n}\right)_{d, n} \in \underset{(n, d)}{\lim _{(1,}} \operatorname{Gal}\left(\Gamma_{d}^{(n)} / K\right)=G_{K}$. Moreover, the injective mapping $\boldsymbol{\phi}_{K}^{(\varphi)}: G_{K} \rightarrow \nabla_{K}^{o}$ is a 1-cocycle, that is, for $\sigma, \tau \in G_{K}$ with respective coherent sequences $\left(\sigma_{d, n}\right)_{d, n},\left(\tau_{d, n}\right)_{d, n} \in \underset{(n, d)}{\lim } \operatorname{Gal}\left(\Gamma_{d}^{(n)} / K\right)$,

$$
\boldsymbol{\phi}_{K}^{(\varphi)}(\sigma \tau)=\phi_{K}^{(\varphi)}(\sigma) \phi_{K}^{(\varphi)}(\tau)^{\sigma} \text {. }
$$


Also the bijective mapping $\boldsymbol{\Phi}_{K}^{(\varphi)}: G_{K} \rightarrow \nabla_{K}$ is a 1-cocycle, i.e., for $\sigma, \tau \in G_{K}$ with respective coherent sequences $\left(\sigma_{d, n}\right)_{d, n},\left(\tau_{d, n}\right)_{d, n} \in \underset{(n, d)}{\lim _{(}} \operatorname{Gal}\left(\Gamma_{d}^{(n)} / K\right)$,

$$
\boldsymbol{\Phi}_{K}^{(\varphi)}(\sigma \tau)=\boldsymbol{\Phi}_{K}^{(\varphi)}(\sigma) \boldsymbol{\Phi}_{K}^{(\varphi)}(\tau)^{\sigma} .
$$

Definition 3.4. The injective 1-cocycle $\phi_{K}^{(\varphi)}: G_{K} \rightarrow \nabla_{K}^{o}$ is called the generalized arrow for $K$, and the bijective 1-cocycle $\boldsymbol{\Phi}_{K}^{(\varphi)}: G_{K} \rightarrow \nabla_{K}$ is called the non-abelian local reciprocity map of $K$.

The 1-cocycles $\boldsymbol{\phi}_{K}^{(\varphi)}$ and $\boldsymbol{\Phi}_{K}^{(\varphi)}$ are related to each other by

$$
\boldsymbol{\Phi}_{K}^{(\varphi)}=c_{K} \circ \boldsymbol{\phi}_{K}^{(\varphi)} .
$$

4. Ramification theory. Now, by Theorems 2.7 and 2.20 of [Ik-Se-2] (cf. also Remark 3.1 in Section 3), the ramification theorems for the generalized arrow $\phi_{\Gamma_{d}^{(n)} / K}$ and for the generalized Fesenko reciprocity map $\boldsymbol{\Phi}_{\Gamma_{d}^{(n)} / K}^{(\varphi)}$ give, for $0 \leq w \in \mathbb{Z}$, the inclusions

$$
\begin{aligned}
& \phi_{\Gamma_{d}^{(n)} / K}^{(\varphi)}\left(\operatorname{Gal}\left(\Gamma_{d}^{(n)} / K\right)_{w}-\operatorname{Gal}\left(\Gamma_{d}^{(n)} / K\right)_{w+1}\right) \\
& \subseteq\left\langle 1_{K^{\times} / N_{K_{d}^{\mathrm{nr}} / K} K_{d}^{\mathrm{nr} \times}}\right\rangle \times\left(\left(U_{\widetilde{\mathbb{X}}\left(\Gamma_{d}^{(n)} / K\right)}^{\diamond}\right)^{w} U_{\mathbb{X}\left(\Gamma_{d}^{(n)} / K\right)} / U_{\mathbb{X}\left(\Gamma_{d}^{(n)} / K\right)}\right. \\
& \left.-\left(U_{\widetilde{\mathbb{X}}\left(\Gamma_{d}^{(n)} / K\right)}\right)^{w+1} U_{\mathbb{X}\left(\Gamma_{d}^{(n)} / K\right)} / U_{\mathbb{X}\left(\Gamma_{d}^{(n)} / K\right)}\right),
\end{aligned}
$$

and

$$
\begin{aligned}
& \boldsymbol{\Phi}_{\Gamma_{d}^{(n)} / K}^{(\varphi)}\left(\operatorname{Gal}\left(\Gamma_{d}^{(n)} / K\right)_{w}-\operatorname{Gal}\left(\Gamma_{d}^{(n)} / K\right)_{w+1}\right)
\end{aligned}
$$

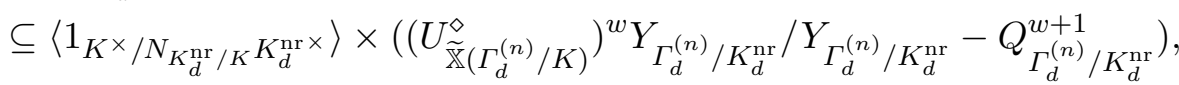

where $\operatorname{Gal}\left(\Gamma_{d}^{(n)} / K\right)_{w}$ denotes the $w$ th higher ramification subgroup in the lower numbering of the Galois group $\operatorname{Gal}\left(\Gamma_{d}^{(n)} / K\right)$ corresponding to the infinite APF-Galois extension $\Gamma_{d}^{(n)} / K$.

The aim of this section is to state and prove ramification theorems for the generalized arrow $\phi_{K}^{(\varphi)}: G_{K} \rightarrow \nabla_{K}^{o}$ and for the non-abelian local reciprocity $\operatorname{map} \boldsymbol{\Phi}_{K}^{(\varphi)}: G_{K} \rightarrow \nabla_{K}$.

\subsection{Higher ramification subgroups of $G_{K}$ in the upper number-} ing. To simplify the discussion, we introduce the following notation.

Notation 4.1. For every $1 \leq d, n \in \mathbb{Z}$, the Galois group $\operatorname{Gal}\left(\Gamma_{d}^{(n)} / K\right)$ is denoted by $G(d, n)$. Moreover, for any $-1 \leq w \in \mathbb{R}, G(d, n)^{w}$ denotes the $w$ th ramification subgroup of $G(d, n)$ in the upper numbering. 
Proposition 4.2. For $\left(n^{\prime}, d^{\prime}\right),(n, d) \in \mathbb{Z}_{\geq 1} \times \mathbb{Z}_{\geq 1}$ satisfying $n^{\prime} \leq n$ and $d^{\prime} \mid d$, and for $0 \leq w \in \mathbb{Z}$,

$$
\psi_{\Gamma_{d^{\prime}}^{\left(n^{\prime}\right)} / K_{d^{\prime}}^{\mathrm{nr}}}(w) \leq \psi_{\Gamma_{d}^{(n)} / K_{d}^{\mathrm{nr}}}(w) .
$$

Proof. As $\Gamma_{d^{\prime}}^{\left(n^{\prime}\right)}$ and $\Gamma_{d}^{(n)}$ are APF-extensions over the field $K$, for every $-1<x \in \mathbb{R}$, setting $G_{\Gamma_{d^{\prime}}^{\left(n^{\prime}\right)}}^{0}=G_{K}^{0} \cap G_{\Gamma_{d^{\prime}}^{\left(n^{\prime}\right)}}$ and $G_{\Gamma_{d}^{(n)}}^{0}=G_{K}^{0} \cap G_{\Gamma_{d}^{(n)}}$, we have $\left(G_{K}^{0}: G_{K}^{x} G_{\Gamma_{d^{\prime}}^{\left(n^{\prime}\right)}}^{0}\right)<\infty$ and $\left(G_{K}^{0}: G_{K}^{x} G_{\Gamma_{d}^{(n)}}^{0}\right)<\infty$ (cf. Fo-Wi-1, Fo-Wi-2, Win]). Now, if $n^{\prime} \leq n$ and $d^{\prime} \mid d$, then $\Gamma_{d^{\prime}}^{\left(n^{\prime}\right)} \subseteq \Gamma_{d}^{(n)}$. Therefore,

$$
\left(G_{K}^{0}: G_{K}^{x} G_{\Gamma_{d^{\prime}}^{\left(n^{\prime}\right)}}^{0}\right) \leq\left(G_{K}^{0}: G_{K}^{x} G_{\Gamma_{d}^{(n)}}^{0}\right)<\infty,
$$

as $G_{\Gamma_{d}^{(n)}} \subseteq G_{\Gamma_{d^{\prime}}^{\left(n^{\prime}\right)}}$. Hence, for $0 \leq w \in \mathbb{Z}$,

$$
\psi_{\Gamma_{d^{\prime}}^{\left(n^{\prime}\right)} / K}(w)=\int_{0}^{w}\left(G_{K}^{0}: G_{K}^{x} G_{\Gamma_{d^{\prime}}^{\left(n^{\prime}\right)}}^{0}\right) d x \leq \int_{0}^{w}\left(G_{K}^{0}: G_{K}^{x} G_{\Gamma_{d}^{(n)}}^{0}\right) d x=\psi_{\Gamma_{d}^{(n)} / K}(w) .
$$

Now, the desired inequality follows, because

$$
\psi_{\Gamma_{d^{\prime}}^{\left(n^{\prime}\right)} / K}(w)=\psi_{\Gamma_{d^{\prime}}^{\left(n^{\prime}\right)} / K_{d^{\prime}}^{\mathrm{nr}}} \circ \psi_{K_{d^{\prime}}^{\mathrm{nr}} / K}(w)=\psi_{\Gamma_{d^{\prime}}^{\left(n^{\prime}\right)} / K_{d^{\prime}}^{\mathrm{nr}}}(w)
$$

and likewise

$$
\psi_{\Gamma_{d}^{(n)} / K}(w)=\psi_{\Gamma_{d}^{(n)} / K_{d}^{\mathrm{nr}}} \circ \psi_{K_{d}^{\mathrm{nr}} / K}(w)=\psi_{\Gamma_{d}^{(n)} / K_{d}^{\mathrm{nr}}}(w) .
$$

Remark 4.3. Note that Proposition 4.2 is more generally true in the following setting. Let $L$ be an infinite APF-Galois extension over $K$ satisfying $K \subset L \subset K_{\varphi^{d}}$ with $\left[\kappa_{L}: \kappa_{K}\right]=d$, and $M / K$ be an infinite Galois sub-extension of $L / K$ satisfying $K \subset M \subset K_{\varphi^{d^{\prime}}}$ with $\left[\kappa_{M}: \kappa_{K}\right]=d^{\prime}$, where $d^{\prime} \mid d$. Then, for $0 \leq w \in \mathbb{Z}$,

$$
\psi_{M / M_{0}}(w) \leq \psi_{L / L_{0}}(w),
$$

where $L_{0}=L \cap K^{\mathrm{nr}}$ and $M_{0}=M \cap K^{\mathrm{nr}}$. The proof follows the same lines.

It is well-known that, for a fixed $-1 \leq w \in \mathbb{R}$, the projective limit

$$
G_{K}^{w}:=\varliminf_{(n, d)}^{\lim } G(d, n)^{w}
$$

over the restriction morphisms

$$
r_{\left(n^{\prime}, d^{\prime}\right)}^{(n, d)}: G(d, n)^{w} \rightarrow G\left(d^{\prime}, n^{\prime}\right)^{w}
$$

for $(n, d),\left(n^{\prime}, d^{\prime}\right) \in \mathbb{Z}_{\geq 1} \times \mathbb{Z}_{\geq 1}$ satisfying $n^{\prime} \leq n$ and $d^{\prime} \mid d$ defines a subgroup $G_{K}^{w}$ of the absolute Galois group $G_{K}$, and we have the following definition.

Definition 4.4. For $-1 \leq w \in \mathbb{R}$, the group $G_{K}^{w}$ is called the $w$ th higher ramification subgroup of $G_{K}$ in the upper numbering. 
However, it turns out that we need a finer upper ramification "filtration" of $G_{K}$. Let $\underline{w}:=\left(w_{(n, d)}\right)$ be a net in $\mathbb{R}_{\geq-1}$ always assumed to be indexed over the directed set $\mathbb{Z}_{\geq 1} \times \mathbb{Z}_{\geq 1}$, where $\left(n^{\prime}, d^{\prime}\right) \preceq(n, d)$ if $n^{\prime} \leq n$ and $d^{\prime} \mid d$ for $(n, d),\left(n^{\prime}, d^{\prime}\right) \in \mathbb{Z}_{\geq 1} \times \mathbb{Z}_{\geq 1}$. Furthermore, assume that the net $\underline{w}$ in $\mathbb{R}_{\geq-1}$ is increasing, that is, $w_{\left(n^{\prime}, d^{\prime}\right)} \leq w_{(n, d)}$ if $\left(n^{\prime}, d^{\prime}\right) \preceq(n, d)$. In case $\underline{w}=\left(w_{(n, d)}\right)$ in $\mathbb{R}_{\geq-1}$ is constant, that is, $w_{(n, d)}=c$ for every $(n, d) \in \mathbb{Z}_{\geq 1} \times \mathbb{Z}_{\geq 1}$, the net $\underline{w}$ will be simply denoted by $c$.

Note that, for an increasing net $\underline{w}$ in $\mathbb{R}_{\geq-1}$, the projective limit

$$
G \frac{w}{K}:=\lim _{(n, d)} G(d, n)^{w_{(n, d)}}
$$

over the restriction morphisms

$$
r_{\left(n^{\prime}, d^{\prime}\right)}^{(n,)^{\prime}}: G(d, n)^{w_{(n, d)}} \rightarrow G\left(d^{\prime}, n^{\prime}\right)^{w_{(n, d)}} \hookrightarrow G\left(d^{\prime}, n^{\prime}\right)^{w_{\left(n^{\prime}, d^{\prime}\right)}}
$$

for $(n, d),\left(n^{\prime}, d^{\prime}\right) \in \mathbb{Z}_{\geq 1} \times \mathbb{Z}_{\geq 1}$ satisfying $n^{\prime} \leq n$ and $d^{\prime} \mid d$ defines a subgroup $G \frac{w}{K}$ of the absolute Galois group $G_{K}$, and we have the following definition.

Definition 4.5. For an increasing net $\underline{w}$ in $\mathbb{R}_{\geq-1}$, the group $G \frac{w}{K}$ is called the $\underline{w}$ th higher ramification subgroup of $G_{K}$ in the upper numbering.

Definition 4.6. Let $\underline{w}=\left(w_{(n, d)}\right)$ be an increasing net in $\mathbb{R}_{\geq-1}$. The net $\underline{w}^{\prime}$ in $\mathbb{R}_{\geq-1}$ defined by

$$
w_{(n, d)}^{\prime}=\varphi_{\Gamma_{d}^{(n)} / K_{d}^{\mathrm{nr}}}\left(\psi_{\Gamma_{d}^{(n)} / K_{d}^{\mathrm{nr}}}\left(w_{(n, d)}\right)+1\right),
$$

for every pair $(n, d)$, which is clearly an increasing net in $\mathbb{R}_{\geq-1}$, is called the successor of $\underline{w}$.

Note that, for any increasing net $\underline{w}$ in $\mathbb{R}_{\geq-1}$, we have the inclusion

$$
G_{K}^{w^{\prime}} \subseteq G \frac{w}{K}
$$

because $G(d, n)_{\Gamma_{d}^{(n)} / K_{d}^{\mathrm{nr}}}\left(w_{(n, d)}\right)+1 \subseteq G(d, n)_{\psi_{\Gamma_{d}^{(n)} / K_{d}^{\mathrm{nn}}}\left(w_{(n, d)}\right)}$ for every pair $(n, d)$. The proof of the following lemma is clear.

Lemma 4.7. For any increasing net $\underline{w}$ in $\mathbb{R}_{\geq-1}$ and for $\sigma=\left(\sigma_{d, n}\right)_{d, n} \in$ $\lim _{\longleftarrow} G(d, n)^{w_{(n, d)}}=G_{K}^{\frac{w}{K}}$, the following two conditions are equivalent. $(n, d)$

(i) $\sigma \in G \frac{w}{K}-G \frac{w^{\prime}}{K}$.

(ii) $\sigma_{d, n} \in G(d, n)_{\psi_{d}^{(n)} / K_{d}^{\mathrm{nn}}}\left(w_{(n, d)}\right)-G(d, n)_{\Gamma_{d}^{(n)} / K_{d}^{\mathrm{nr}}}\left(w_{(n, d)}\right)+1$ for some $(n, d) \in \mathbb{Z}_{\geq 1} \times \mathbb{Z}_{\geq 1}$.

4.2. The groups $\nabla_{K}^{o, w}$ and $\nabla \frac{w}{K}$ for an increasing net $\underline{w}$ in $\mathbb{R}_{\geq-1}$. The following proposition is central to what follows.

Proposition 4.8. Let $L$ be an infinite APF-Galois extension over $K$ satisfying $K \subset L \subset K_{\varphi^{d}}$ with $\left[\kappa_{L}: \kappa_{K}\right]=d$, and $M / K$ be an infinite Galois 
sub-extension of $L / K$ satisfying $K \subset M \subset K_{\varphi^{d^{\prime}}}$ with $\left[\kappa_{M}: \kappa_{K}\right]=d^{\prime}$, where $d^{\prime} \mid d$. Then

$$
\widetilde{\mathcal{N}}_{L / M} \circ\langle\varphi\rangle_{L / M}\left(\left(U_{\widetilde{\mathbb{X}}(L / K)}^{\diamond}\right)^{w}\right) \subseteq\left(U_{\widetilde{\mathbb{X}}(M / K)}^{\diamond}\right)^{w}
$$

for every $0 \leq w \in \mathbb{Z}$.

Proof. For $0 \leq w \in \mathbb{Z}$, let $\alpha=\left(\alpha_{\widetilde{E}_{i}}\right)_{0 \leq i \in \mathbb{Z}} \in\left(U_{\widetilde{\mathbb{X}}(L / K)}^{\diamond}\right)^{w}$. That is, the norm coherent sequence $\alpha=\left(\alpha_{\widetilde{E}_{i}}\right)_{0 \leq i \in \mathbb{Z}} \in U_{\widetilde{\mathbb{X}}(L / K)}$ satisfies

$$
\nu_{\widetilde{\mathbb{X}}(L / K)}\left(\left(\alpha_{\widetilde{E}_{i}}\right)_{0 \leq i \in \mathbb{Z}}-1_{\widetilde{\mathbb{X}}(L / K)}\right)=\nu_{\widetilde{K}}\left(\alpha_{\widetilde{K}}-1\right) \geq w,
$$

where the equality follows from the definition of addition on $\widetilde{\mathbb{X}}(L / K)$ and the valuation $v_{\widetilde{\mathbb{X}}(L / K)}$ on $\widetilde{\mathbb{X}}(L / K)$. Thus, by the definition of the mapping $\tilde{\mathcal{N}}_{L / M} \circ\langle\varphi\rangle_{L / M}: \mathbb{X}(L / K)^{\times} \rightarrow \mathbb{X}(M / K)^{\times}$, it follows that

$$
\begin{aligned}
& \nu_{\widetilde{\mathbb{X}}(M / K)}\left(\widetilde{\mathcal{N}}_{L / M} \circ\langle\varphi\rangle_{L / M}\left(\left(\alpha_{\widetilde{E}_{i}}\right)_{0 \leq i \in \mathbb{Z}}\right)-1_{\widetilde{\mathbb{X}}(M / K)}\right) \\
& \quad=\nu_{\widetilde{K}}\left(\alpha_{\widetilde{K}}^{\left(1+\varphi^{d^{\prime}}+\cdots+\varphi^{d^{\prime}(f(L / M)-1)}\right)^{2}}-1\right)=\nu_{\widetilde{K}}\left(\alpha_{\widetilde{K}}^{f(L / M)^{2}}-1\right),
\end{aligned}
$$

as $\alpha=\left(\alpha_{\widetilde{E}_{i}}\right)_{0 \leq i \in \mathbb{Z}} \in U_{\widetilde{\mathbb{X}}(L / K)}^{\diamond}$ and the $\widetilde{K}$-coordinate of $\alpha$ satisfies $\alpha_{\widetilde{K}} \in U_{L_{0}}$, where $L_{0}=L \cap K^{\mathrm{nr}}$. Thus,

$$
\begin{aligned}
\nu_{\widetilde{\mathbb{X}}(M / K)}\left(\widetilde{\mathcal{N}}_{L / M} \circ\langle\varphi\rangle_{L / M}\left(\left(\alpha_{\widetilde{E}_{i}}\right)_{0 \leq i \in \mathbb{Z}}\right)-1_{\widetilde{\mathbb{X}}(M / K)}\right) \\
=\nu_{\widetilde{K}}\left(\alpha_{\widetilde{K}}-1\right)+\nu_{\widetilde{K}}\left(\sum_{0 \leq \ell \leq f(L / M)^{2}} \alpha_{\widetilde{K}}^{\ell}\right) \geq w,
\end{aligned}
$$

which shows that $\widetilde{\mathcal{N}}_{L / M} \circ\langle\varphi\rangle_{L / M}\left(\left(\alpha_{\widetilde{E}_{i}}\right)_{0 \leq i \in \mathbb{Z}}\right) \in U_{\widetilde{\mathbb{X}}(M / K)}^{w}$. Combining this with the property (ii) of equation (2.21) in [Ik-Se-2] yields the assertion.

Notation 4.9. Let $L$ be an infinite APF-Galois extension over $K$ satisfying $K \subset L \subset K_{\varphi^{d}}$ with $\left[\kappa_{L}: \kappa_{K}\right]=d$. For $0 \leq w \in \mathbb{R}$, let

$$
\begin{aligned}
& \left(\nabla_{L / K}^{(\varphi), o}\right)^{w}=\nabla_{L / K}^{o, w}=K^{\times} / N_{L_{0} / K} L_{0}^{\times} \times\left(U_{\widetilde{\mathbb{X}}(L / K)}^{\diamond}\right)^{w} U_{\mathbb{X}(L / K)} / U_{\mathbb{X}(L / K)}, \\
& \left(\nabla_{L / K}^{(\varphi)}\right)^{w}=\nabla_{L / K}^{w}=K^{\times} / N_{L_{0} / K} L_{0}^{\times} \times\left(U_{\widetilde{\mathbb{X}}(L / K)}^{\diamond}\right)^{w} Y_{L / L_{0}} / Y_{L / L_{0}} .
\end{aligned}
$$

Therefore, by Remark 4.3 , for the local fields $L$ and $M$ as in Proposition 4.8, and for $0 \leq w_{L / K}, w_{M / K} \in \mathbb{R}$ satisfying $w_{M / K} \leq w_{L / K}$, the map $\mathcal{C}_{L / M}^{o}$ introduced in Notation 3.3(i) restricts to

$$
\mathcal{C}_{L / M}^{o}: \nabla_{L / K}^{o, \psi_{L / L_{0}}\left(w_{L / K}\right)} \rightarrow \nabla_{M / K}^{o, \psi_{M / M_{0}}\left(w_{M / K}\right)},
$$

and the map $\mathcal{C}_{L / M}$ introduced in Notation 3.3(ii) restricts to

$$
\mathcal{C}_{L / M}: \nabla_{L / K}^{\psi_{L / L_{0}}\left(w_{L / K}\right)} \rightarrow \nabla_{M / K}^{\psi_{M / M_{0}}\left(w_{M / K}\right)} .
$$

Thus, the following corollary follows directly. 
Corollary 4.10. For an increasing net $\underline{w}=\left(w_{(n, d)}\right)$ in $\mathbb{R}_{\geq 0}$, the systems

$$
\left\{\nabla_{\Gamma_{d}^{(n)} / K}^{o, \Gamma_{d}^{(n)} / K_{d}^{\mathrm{nr}}\left(w_{(n, d)}\right)} ; \mathcal{C}_{\Gamma_{d}^{(n)} / \Gamma_{d^{\prime}}^{\left(n^{\prime}\right)}}^{o}\right\}_{\substack{n^{\prime} \leq n \\ d^{\prime} \mid d}}
$$

and

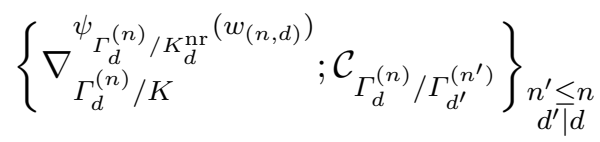

are projective.

Proof. Follows from the projectivity of the systems (3.22) and (3.23), and from Proposition 4.8 combined with Proposition 4.2 ,

For any increasing net $\underline{w}$ in $\mathbb{R}_{\geq 0}$, let

$$
\begin{aligned}
& \left(\nabla_{K}^{(\varphi), o}\right)^{\underline{w}}:=\nabla_{K}^{o, w}={\underset{\lim }{(n, d)}}_{\nabla_{d}^{o, \psi} / K}^{o(n) / K_{d}^{(n)}\left(w_{(n, d)}\right)}
\end{aligned}
$$

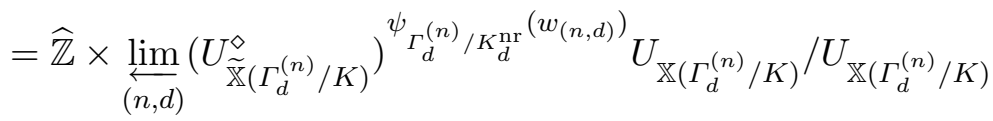

and

$$
\begin{aligned}
& \left(\nabla_{K}^{(\varphi)}\right)^{\underline{w}}:=\nabla_{K}^{\frac{w}{K}}={\underset{\leftarrow}{(n, d)}}_{\lim _{d}} \nabla_{\Gamma_{d}^{(n)} / K}^{\psi_{d}^{(n)} / K}
\end{aligned}
$$

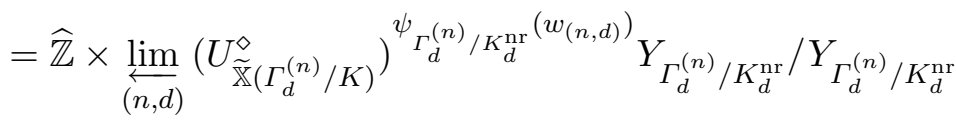

be the projective limits of the systems 4.11) and (4.12) respectively. These limits $\left(\nabla_{K}^{(\varphi), o}\right)^{\underline{w}}$ and $\left(\nabla_{K}^{(\varphi)}\right)^{\underline{w}}$, or $\nabla_{K}^{o, \underline{w}}$ and $\nabla_{K}^{\frac{w}{K}}$ if there is no risk of confusion, depend on the choice of a Lubin-Tate splitting $\varphi$ over $K$.

Lemma 4.11. For $(n, d),\left(n^{\prime}, d^{\prime}\right)$ satisfying $n^{\prime} \leq n, d^{\prime} \mid d$, and for $0 \leq$ $w_{(n, d)}, w_{\left(n^{\prime}, d^{\prime}\right)} \in \mathbb{R}$ satisfying $w_{\left(n^{\prime}, d^{\prime}\right)} \leq w_{(n, d)}$ and $0 \leq \psi_{\Gamma_{d}^{(n)} / K}\left(w_{(n, d)}\right)$, $\psi_{\Gamma_{d^{\prime}}^{\left(n^{\prime}\right)} / K}\left(w_{\left(n^{\prime}, d^{\prime}\right)}\right) \in \mathbb{Z}$, the squares

$$
\begin{aligned}
& G(d, n)^{w_{(n, d)}} \stackrel{\phi_{\Gamma_{d}^{(n)} / K}^{(\varphi)}}{\longrightarrow} A_{(n, d)}^{(U)}
\end{aligned}
$$

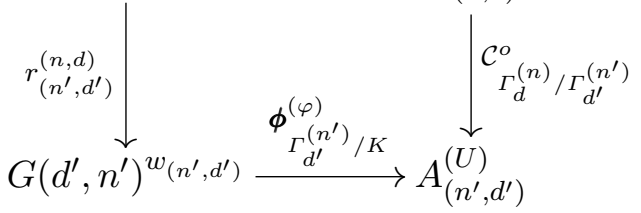


and

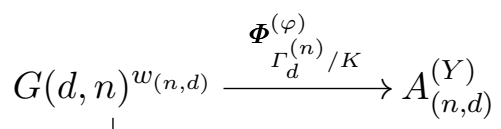

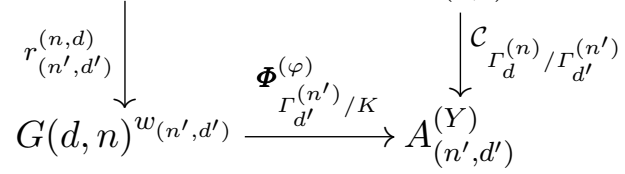

are commutative, where for $(n, d) \in \mathbb{Z}_{\geq 1} \times \mathbb{Z}_{\geq 1}$ and $0 \leq w_{(n, d)} \in \mathbb{R}$ satisfying $0 \leq \psi_{\Gamma_{d}^{(n)} / K}\left(w_{(n, d)}\right) \in \mathbb{Z}$

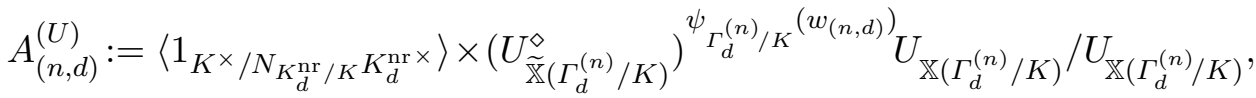

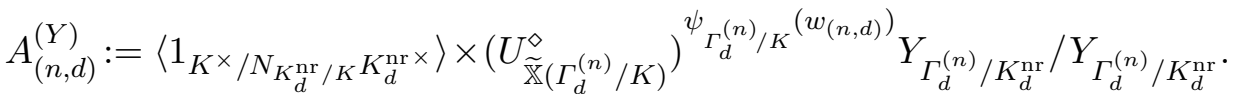

Proof. Follows from Proposition 4.2, and the basic property (iii) of ramification theory of $\boldsymbol{\phi}_{L / K}^{(\varphi)}$ and $\boldsymbol{\Phi}_{L / K}^{(\varphi)}$ together with the basic property (i) of $\boldsymbol{\phi}_{L / K}^{(\varphi)}$ and $\boldsymbol{\Phi}_{L / K}^{(\varphi)}$ stated in Section 3 .

For any increasing net $\underline{w}=\left(w_{(n, d)}\right)$ in $\mathbb{R}_{\geq 0}$, let ${ }_{1} \nabla_{K}^{o, \underline{w}}$ denote the kernel of the projection $\operatorname{Pr}_{1}: \nabla_{K}^{o, \underline{w}} \rightarrow \widehat{\mathbb{Z}}$, and ${ }_{1} \nabla \frac{w}{K}$ denote the kernel of the projection $\operatorname{Pr}_{1}: \nabla \frac{w}{K} \rightarrow \widehat{\mathbb{Z}}$. An immediate consequence of Lemma 4.11 is

Corollary 4.12. Let $\underline{w}$ be any increasing net in $\mathbb{R}_{\geq 0}$ and $\sigma \in G \frac{w}{K}$. Then:

(i) $\boldsymbol{\phi}_{K}^{(\varphi)}(\sigma) \in{ }_{1} \nabla_{K}^{o, \underline{w}}$.

(ii) $\boldsymbol{\Phi}_{K}^{(\varphi)}(\sigma) \in{ }_{1} \nabla \frac{w}{K}$.

Now, for an increasing net $\underline{w}=\left(w_{(n, d)}\right)$ in $\mathbb{R}_{\geq 0}$, introduce

$$
Q \frac{w}{K}=c_{K}\left({ }_{1} \nabla_{K}^{o, \underline{w}} \cap \operatorname{im}\left(\phi_{K}^{(\varphi)}\right)\right),
$$

where $c_{K}: \nabla_{K}^{o} \rightarrow \nabla_{K}$ is the canonical map defined by (3.28. Note that $c_{K}\left({ }_{1} \nabla_{K}^{o, w}\right)={ }_{1} \nabla_{K}^{w}$ by the commutativity of the square 3.27 and by Propositions 4.2 and 4.8 .

LEMMA 4.13. For an increasing net $\underline{w}=\left(w_{(n, d)}\right)$ in $\mathbb{R}_{\geq 0}$,

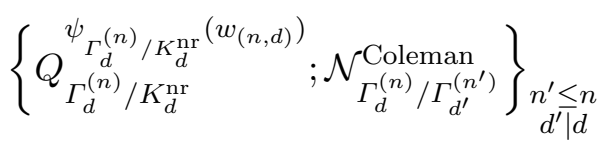

is a projective system and its projective limit is

$$
Q \frac{w}{K}=\left\langle 1_{\widehat{\mathbb{Z}}}\right\rangle \times{\underset{(\lim }{\overleftarrow{(n, d)}}}_{Q_{\Gamma_{d}^{(n)} / K_{d}^{\mathrm{nr}}}^{\psi}}^{\psi_{\Gamma_{(n)}^{(n)} / K_{\mathrm{nr}}}^{\left(w^{\prime}\right.}} .
$$


Proof. The projectivity of 4.18 follows from the projectivity of the

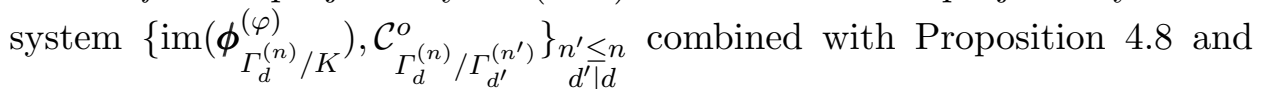
(3.27). Moreover, the equality (4.19) follows from (3.28) and (3.27).

In the lemma below, whose proof is clear, $\underline{w}^{\prime}$ denotes the successor of $\underline{w}$.

Lemma 4.14. (i) For any increasing net $\underline{w}=\left(w_{(n, d)}\right)$ in $\mathbb{R}_{\geq-1}$ and for an element $u=\left(u_{d, n}\right)_{d, n}=\left(\left(1_{K^{\times}} / N_{K_{d}^{\mathrm{nr}} / K} K_{d}^{\mathrm{nr} \times}, \bar{U}_{d, n}\right)\right)_{d, n}$ of

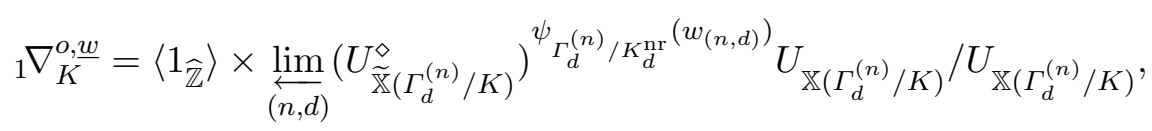

we have: $u \in{ }_{1} \nabla_{K}^{o, \underline{w}}-{ }_{1} \nabla_{K}^{o, \underline{w^{\prime}}}$ if and only if

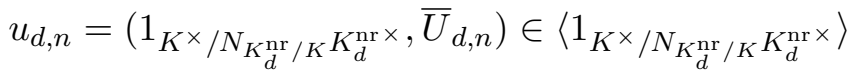

$$
\begin{aligned}
& \times\left(\left(U_{\widetilde{\mathbb{X}}\left(\Gamma_{d}^{(n)} / K\right)}^{\diamond}\right)^{\psi_{d}^{(n)} / K_{d}^{\mathrm{nr}}\left(w_{(n, d)}\right)} U_{\mathbb{X}\left(\Gamma_{d}^{(n)} / K\right)} / U_{\mathbb{X}\left(\Gamma_{d}^{(n)} / K\right)}\right. \\
& \left.-\left(U_{\widetilde{\mathbb{X}}\left(\Gamma_{d}^{(n)} / K\right)}\right)^{\psi_{\Gamma_{d}^{(n)} / K_{d}^{\mathrm{nr}}}\left(w_{(n, d)}\right)+1} U_{\mathbb{X}\left(\Gamma_{d}^{(n)} / K\right)} / U_{\mathbb{X}\left(\Gamma_{d}^{(n)} / K\right)}\right)
\end{aligned}
$$

for some $(n, d) \in \mathbb{Z}_{\geq 1} \times \mathbb{Z}_{\geq 1}$.

(ii) For any increasing net $\underline{w}=\left(w_{(n, d)}\right)$ in $\mathbb{R}_{\geq-1}$ and for an element

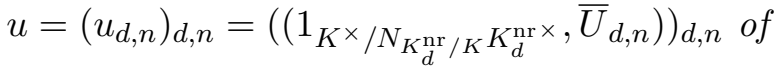

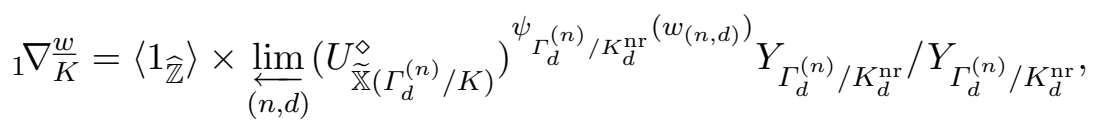

we have: $u \in{ }_{1} \nabla \frac{w}{K}-Q \frac{w^{\prime}}{K}$ if and only if

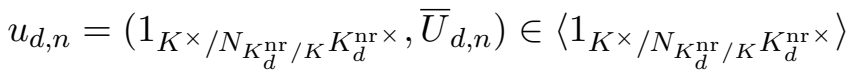

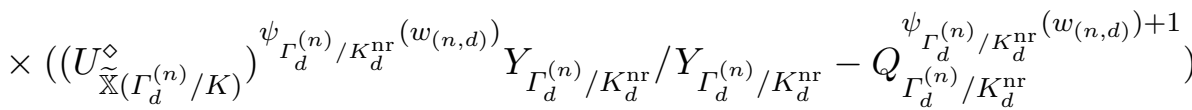

for some $(n, d) \in \mathbb{Z}_{\geq 1} \times \mathbb{Z}_{\geq 1}$.

4.3. Main theorems. We can now state and prove the main theorem, that is, the ramification theorem for the non-abelian local reciprocity map $\boldsymbol{\Phi}_{K}^{(\varphi)}$. In order to do so, we first prove the ramification theorem for the generalized arrow $\phi_{K}^{(\varphi)}: G_{K} \rightarrow \nabla_{K}^{o}$.

THEOREM 4.15 (Ramification theorem for $\left.\boldsymbol{\phi}_{K}^{(\varphi)}\right)$. For any increasing net $\underline{w}=\left(w_{(n, d)}\right)$ in $\mathbb{R}_{\geq-1}$ satisfying $0 \leq \psi_{\Gamma_{d}^{(n)} / K_{d}^{\mathrm{nr}}}\left(w_{(n, d)}\right) \in \mathbb{Z}$ for every $(n, d)$ 
in $\mathbb{Z}_{\geq 1} \times \mathbb{Z}_{\geq 1}$, we have the inclusion

$$
\phi_{K}^{(\varphi)}\left(G \frac{w}{K}-G \frac{w^{\prime}}{K}\right) \subseteq{ }_{1} \nabla_{K}^{o, \underline{w}}-{ }_{1} \nabla_{K}^{o, w^{\prime}} .
$$

Proof. Let $\underline{w}$ be as in the assumptions. Let

$$
\sigma=\left(\sigma_{d, n}\right)_{d, n} \in \lim _{(n, d)} G(d, n)^{w_{(n, d)}}=G_{K}^{\frac{w}{K}} .
$$

Clearly, by Corollary $4.12(\mathrm{i}), \boldsymbol{\phi}_{K}^{(\varphi)}(\sigma) \in{ }_{1} \nabla_{K}^{o, \underline{w}}$. By Lemma 4.7, the condition $\sigma \in G \frac{w}{K}-G \frac{w^{\prime}}{K}$ is equivalent to the existence of a pair $(n, d) \in \mathbb{Z}_{\geq 1} \times \mathbb{Z}_{\geq 1}$ satisfying $\sigma_{d, n} \in G(d, n)_{\Gamma_{d}^{(n)} / K_{d}^{\mathrm{nr}}}\left(w_{(n, d)}\right)-G(d, n)_{\Gamma_{d}^{(n)} / K_{d}^{\mathrm{nr}}}\left(w_{(n, d)}\right)+1$. Therefore, by the ramification theorem for the generalized arrow $\phi_{\Gamma_{d}^{(n)} / K}^{(\varphi)}$, stated in (4.1),

$$
\begin{aligned}
& \boldsymbol{\phi}_{\Gamma_{d}^{(n)} / K}^{(\varphi)}\left(\sigma_{d, n}\right) \in\left\langle 1_{\left.K^{\times} / N_{K_{d}^{\mathrm{nr}} / K} K_{d}^{\mathrm{nr} \times}\right\rangle}\right\rangle \\
& \times\left(\left(U_{\widetilde{\mathbb{X}}\left(\Gamma_{d}^{(n)} / K\right)}^{\diamond}\right)^{\psi_{d}^{(n)} / K_{d}^{\mathrm{nr}}\left(w_{(n, d)}\right)} U_{\mathbb{X}\left(\Gamma_{d}^{(n)} / K\right)} / U_{\mathbb{X}\left(\Gamma_{d}^{(n)} / K\right)}\right. \\
& \left.-\left(U_{\widetilde{\mathbb{X}}\left(\Gamma_{d}^{(n)} / K\right)}\right)^{\psi_{d}^{(n)} / K_{d}^{\mathrm{nr}}\left(w_{(n, d)}\right)+1} U_{\mathbb{X}\left(\Gamma_{d}^{(n)} / K\right)} / U_{\mathbb{X}\left(\Gamma_{d}^{(n)} / K\right)}\right),
\end{aligned}
$$

which proves, by Lemma 4.14(i), that

$$
\phi_{K}^{(\varphi)}(\sigma) \in{ }_{1} \nabla_{K}^{o, \underline{w}}-{ }_{1} \nabla_{K}^{o, \underline{w}^{\prime}} .
$$

THEOREM 4.16 (Ramification theorem for $\left.\boldsymbol{\Phi}_{K}^{(\varphi)}\right)$. For any increasing net $\underline{w}=\left(w_{(n, d)}\right)$ in $\mathbb{R}_{\geq-1}$ satisfying $0 \leq \psi_{\Gamma_{d}^{(n)} / K_{d}^{\mathrm{nr}}}\left(w_{(n, d)}\right) \in \mathbb{Z}$ for every $(n, d) \in$ $\mathbb{Z}_{\geq 1} \times \mathbb{Z}_{\geq 1}$, we have the inclusion

$$
\boldsymbol{\Phi}_{K}^{(\varphi)}\left(G \frac{w}{K}-G \frac{w^{\prime}}{K}\right) \subseteq{ }_{1} \nabla \frac{w}{K}-Q \frac{w^{\prime}}{K} .
$$

Proof. Let $\underline{w}$ be as above. Let $\sigma=\left(\sigma_{d, n}\right)_{d, n} \in \underset{(n, d)}{\lim _{(1,}} G(d, n)^{w_{(n, d)}}=G_{K}^{\frac{w}{K}}$. Clearly, by Corollary 4.12 (ii), $\boldsymbol{\Phi}_{K}^{(\varphi)}(\sigma) \in{ }_{1} \nabla \frac{w}{K}$. By Lemma 4.7 , the condition $\sigma \in G \frac{w}{K}-G \frac{w^{\prime}}{K}$ is equivalent to the existence of a pair $(n, d) \in \mathbb{Z}_{\geq 1} \times \mathbb{Z}_{\geq 1}$ satisfying $\sigma_{d, n} \in G(d, n)_{\Gamma_{d}^{(n)} / K_{d}^{\mathrm{nr}}\left(w_{(n, d)}\right)}-G(d, n)_{\Gamma_{d}^{(n)} / K_{d}^{\mathrm{nr}}}\left(w_{(n, d)}\right)+1$. Therefore, by the ramification theorem for the generalized Fesenko reciprocity $\operatorname{map} \boldsymbol{\Phi}_{\Gamma_{d}^{(n)} / K}^{(\varphi)}$, stated in 4.2),

$$
\begin{aligned}
& \boldsymbol{\Phi}_{\Gamma_{d}^{(n)} / K}^{(\varphi)}\left(\sigma_{d, n}\right) \in\left\langle 1_{\left.K^{\times} / N_{K_{d}^{\mathrm{nr}} / K} K_{d}^{\mathrm{nr} \times}\right\rangle}\right. \\
& \quad \times\left(\left(U_{\widetilde{\mathbb{X}}\left(\Gamma_{d}^{(n)} / K\right)}^{\diamond}\right)^{\psi_{d}^{(n)} / K_{d}^{\mathrm{nr}}\left(w_{(n, d)}\right)} Y_{\Gamma_{d}^{(n)} / K_{d}^{\mathrm{nr}}} / Y_{\Gamma_{d}^{(n)} / K_{d}^{\mathrm{nr}}}-Q_{\Gamma_{d}^{(n)} / K_{d}^{\mathrm{nr}}}^{\psi_{\Gamma^{(n)} / K^{\mathrm{nr}}}\left(w_{(n, d)}\right)+1}\right),
\end{aligned}
$$


which proves, by Lemma 4.14(ii), that

$$
\boldsymbol{\Phi}_{K}^{(\varphi)}(\sigma) \in{ }_{1} \nabla \frac{w}{K}-Q \frac{w^{\prime}}{K}
$$

Acknowledgements. The first named author (K. I. I.) is partly supported by the TÜBİTAK grant $107 \mathrm{~T} 728$.

\section{References}

[Fes-1] I. B. Fesenko, Local reciprocity cycles, in: Invitation to Higher Local Fields, I. B. Fesenko and M. Kurihara (eds.), Geom. Topol. Monogr. 3, Coventry, 2000, 293-298.

[Fes-2] - Nonabelian local reciprocity maps, in: Class Field Theory-Its Centenary and Prospect (Tokyo, 1998), K. Miyake (ed.), Adv. Stud. Pure Math. 30, Math. Soc. Japan, 2001, 63-78.

[Fes-3] - On the image of noncommutative local reciprocity map, Homology Homotopy Appl. 7 (2005), 53-62.

[Fe-Vo] I. B. Fesenko and S. V. Vostokov, Local Fields and Their Extensions, 2nd ed., Transl. Math. Monogr. 121, Amer. Math. Soc., Providence, RI, 2002.

[Fo-Wi-1] J.-M. Fontaine et J.-P. Wintenberger, Le "corps des normes" de certaines extensions algébriques de corps locaux, C. R. Acad. Sci. Paris Sér. A Math. 288 (1979), 367-370.

[Fo-Wi-2] -, 一, Extensions algébriques et corps des normes des extensions APF des corps locaux, ibid. 288 (1979), 441-444.

[Ik-Se-1] K. I. Ikeda and E. Serbest, Fesenko reciprocity map, Algebra i Analiz 20 (2008), no. 3, 112-162 (in Russian).

[Ik-Se-2] —, —, Generalized Fesenko reciprocity map, ibid. 20 (2008), no. 4, 118-159 (in Russian).

[Ik-Se-3] - - - Non-abelian local reciprocity law, Manuscripta Math., to appear.

[Ko-dS] H. Koch and E. de Shalit, Metabelian local class field theory, J. Reine Angew. Math. 478 (1996), 85-106.

[Win] J.-P. Wintenberger, Le corps des normes de certaines extensions infinies de corps locaux; applications, Ann. Sci. École Norm. Sup. 46 (1983), 59-89.

Kâzim Ilhan Ikeda, Erol Serbest

Department of Mathematics

Yeditepe University

İnönü Mah., Kayışdağı Cad.

26 Ağustos Yerleşimi

34755 Kadıköy, Istanbul, Turkey

E-mail: ilhan.ikeda@yeditepe.edu.tr

erol.serbest@yeditepe.edu.tr

Received on 20.11.2009

and in revised form on 24.3.2010 